\title{
Geometric Bäcklund-Darboux Transformations for the KP Hierarchy
}

\author{
By
}

\author{
Gerard F. HeLmincK* and Johan W. van de LeuR**
}

\begin{abstract}
In this paper it is shown that, if you have two planes in the Sato Grassmannian that have an intersection of finite codimension, then the corresponding solutions of the KP hierarchy are linked by Bäcklund-Darboux (shortly BD-)transformations. The pseudodifferential operator that performs this transformation is shown to be built up in a geometric way from so-called elementary BD-transformations and is given here in a closed form. The corresponding action on the tau-function, associated to a plane in the Grassmannian, is also determined
\end{abstract}

\section{$\S 1$. The KP-Hierarchy}

The KP-hierarchy consists of a tower of nonlinear evolution equations in infinitely many variables $\left\{t_{n} \mid n \geq 1\right\}$. It is named after the simplest nontrivial equation in this tower, the Khadomtsev-Petviashvili equation:

$$
\frac{3}{4} \frac{\partial^{2} u}{\partial t_{2}^{2}}=\frac{\partial}{\partial t_{1}}\left(\frac{\partial u}{\partial t_{3}}-3 u \frac{\partial u}{\partial t_{1}}-\frac{1}{4} \frac{\partial^{3} u}{\partial t_{1}^{3}}\right) .
$$

The compact form in which these equations are usually presented, is the socalled Lax form. To give some insight in this form and to formulate precisely

Communicated by M. Kashiwara, September 21, 1998. Revised March 26, 2001.

2000 Mathematics Subject Classification(s): 22E65, 22E67, 22E70, 35Q53, 35Q58, 58B37, 81R10.

* Faculty of Mathematical Sciences, University of Twente, P.O.Box 217, 7500 AE Enschede, The Netherlands.

e-mail: helminck@math.utwente.nl

*** Mathematical Institute, University of Utrecht, P.O. Box 80010, 3508 TA Utrecht, The Netherlands.

e-mail: vdleur@math.uu.nl

Part of this research was done while JvdL was working at the University of Twente, supported by the Netherlands Organization for Scientific Research (NWO). 
what we want, we recall shortly its underlying algebraic structure, see [25] or [24]. Consider the collection of commuting unknowns $\left\{u_{j}^{(i)} \mid j \geq 1, i \geq 0\right\}$ and let $A$ be the ring $\mathbb{C}\left[u_{j}^{(i)}\right]$. On the algebra $A$ we can define a $\mathbb{C}$-linear derivation $\tilde{\partial}: A \rightarrow A$ by putting $\tilde{\partial}\left(u_{j}^{(i)}\right)=u_{j}^{(i+1)}$ for all $i$ and $j$. In the $\operatorname{ring} A[\tilde{\partial}]$ of differential operators in $\tilde{\partial}$ with coefficients from $A$, one can, in general, not take roots of monic operators. Thereto one passes to the extension $A\left[\tilde{\partial}, \tilde{\partial}^{-1}\right)$ of all pseudodifferential operators in $\tilde{\partial}$ with coefficients from $A$. It consists of all expressions

$$
\sum_{i=-\infty}^{N} a_{i} \tilde{\partial}^{i}, a_{i} \in A \text { for all } i
$$

that are added in an obvious way and that are multiplied according to

$$
\tilde{\partial} \cdot a \tilde{\partial}^{i}=\sum_{k=0}^{\infty}\left(\begin{array}{l}
j \\
k
\end{array}\right) \tilde{\partial}^{k}(a) \tilde{\partial}^{i+j-k} .
$$

Each operator $A=\sum a_{j} \tilde{\partial}^{j}$ decomposes as $A=A_{+}+A_{-}$with $A_{+}=\sum_{j \geq 0} a_{j} \tilde{\partial}^{j}$ its differential operator part and $A_{-}=\sum_{j<0} a_{j} \tilde{\partial}^{j}$ its pure integral operator part. The residue of $A$ is the coefficient $a_{-1}$ and we denote it as $\operatorname{Res}_{\tilde{\partial}} A$. Note that in order to define a derivation of $A$ that commutes with $\tilde{\partial}$, it suffices to prescribe the image of the $\left\{u_{j}^{(0)} \mid j \geq 1\right\}$ and this can be done freely. The choice we are interested in here can easily be formulated as an operator equality in $A\left[\tilde{\partial}, \tilde{\partial}^{-1}\right)$. For, let $\tilde{L}$ in $A\left[\tilde{\partial}, \tilde{\partial}^{-1}\right)$ be given by

$$
\tilde{L}=\tilde{\partial}+\sum_{j>0} u_{j}^{(0)} \tilde{\partial}^{-j}
$$

then there is for all $n \geq 1$, a unique derivation $\mathbb{C}$-linear $\tilde{\partial}_{n}: A \rightarrow A$ that commutes with $\tilde{\partial}$ and satisfies

$$
\tilde{\partial}_{n}(\tilde{L}):=0 \tilde{\partial}+\sum_{j>0} \tilde{\partial}_{n}\left(u_{j}^{(0)}\right) \tilde{\partial}^{-j}=\left[(\tilde{L})_{+}^{n}, \tilde{L}\right] .
$$

The derivations $\tilde{\partial}_{n}$ commute among each other (see [25]). This system of equations is called the KP-hierarchy for a reason that will become clear later on. Since the introduction of the derivations $\tilde{\partial}_{n}$ is merely a formal affair, one is interested in concrete realizations of the relations in (1.1). This means that we are looking for commutative $\mathbb{C}$-algebras $R$ equiped with a privileged $\mathbb{C}$-linear 
derivation $\partial: R \rightarrow R$ and a collection of $\mathbb{C}$-linear derivations $\left\{\partial_{n}, n \geq 1\right\}$ commuting with $\partial$. Further there should be a $\mathbb{C}$-algebra morphism $\alpha: A \rightarrow R$ that is compatible with all these derivations i.e. it should satisfy

$$
\alpha \circ \tilde{\partial}=\partial \circ \alpha \quad \text { and } \quad \alpha \circ \tilde{\partial}_{n}=\partial_{n} \circ \alpha, \quad n \geq 1 .
$$

In the present paper, the derivation $\partial$ will be differentiation w.r.t. a parameter $x$ and likewise $\partial_{n}$ will be $\partial / \partial t_{n}$ with $t_{n}$ some parameter and $R$ will be a subring of $\mathbb{C}\left(\left(x, t_{1}, t_{2}, \ldots\right)\right)$. From the first property in (1.2) follows that such an $\alpha$ is completely determined by all the $\left\{l_{j}:=\alpha\left(u_{j}^{(0)}\right), j \geq 1\right\}$ and in order that this property holds, one can choose the $\left\{l_{j}\right\}$ freely. To get the remaining properties of (1.2) it suffices that the Lax operator

$$
L=\partial+\sum_{j>0} l_{j} \partial^{-j}
$$

satisfies the following system of nonlinear equations inside $R\left[\partial, \partial^{-1}\right)$

$$
\partial_{n}(L):=\sum_{j>0} \partial_{n}\left(l_{j}\right) \partial^{-j}=\left[\left(L^{n}\right)_{+}, L\right] \text { for all } n \geq 1 .
$$

The system of equations (1.4) can easily be shown to be equivalent to the so-called Zakharov-Shabat equations

$$
\partial_{n}\left(L^{m}\right)_{+}-\partial_{m}\left(L^{n}\right)_{+}=\left[\left(L^{n}\right)_{+},\left(L^{m}\right)_{+}\right] m, n \geq 1 .
$$

The case $n=2$ and $m=3$ of the system (1.5) implies that $l_{1}$ satisfies the KP-equation and this clarifies the name KP-hierarchy. The formulation (1.4) is called its Lax form. A Lax operator $L$ in $R\left[\partial, \partial^{-1}\right.$ ) satisfying the equations in (1.4) is called a solution of the KP-hierarchy in $R$. Note that for $n=1$ equation (1.4) requires simply that $\partial\left(l_{j}\right)=\partial_{1}\left(l_{j}\right)$ for all $j \geq 1$.

The KP-hierarchy has various interesting subsystems. The ones we are interested in, correspond to the case that $L$ is the $m$-th root of a differential operator $\mathcal{L}$ in $R[\partial]$ of order $m$, i.e. $L^{m}=\left(L^{m}\right)_{+}=\mathcal{L}$. It is called the $m$-th Gelfand-Dickey hierarchy. For $m=2$, this operator $\mathcal{L}$ is the Schrödinger operator $\partial^{2}+2 l_{1}$ and the simplest nontrivial equation is the KdV-equation. Therefore, the second Gelfand-Dickey hierarchy is mostly called the KdV-hierarchy. For the Schrödinger operators

$$
\mathcal{L}=\partial^{2}+2 u
$$

Darboux considered the following transformation: take a non-zero $\phi$ such that

$$
\mathcal{L}(\phi)=\partial^{2}(\phi)+2 u \phi=0
$$


Consider then the new Schrödinger operator

$$
\tilde{\mathcal{L}}=\partial^{2}+2 \tilde{u}, \quad \text { with } \quad \tilde{u}=-u-\frac{\partial(\phi)}{\phi} .
$$

One easily verifies that $\tilde{\mathcal{L}}$ and $\mathcal{L}$ decompose as follows:

$$
\mathcal{L}=\left(\partial+\frac{\partial(\phi)}{\phi}\right)\left(\partial-\frac{\partial(\phi)}{\phi}\right) \text { and } \tilde{\mathcal{L}}=\left(\partial-\frac{\partial(\phi)}{\phi}\right)\left(\partial+\frac{\partial(\phi)}{\phi}\right)
$$

Since $\phi^{-1} \partial \phi=\partial+\partial(\phi) \phi^{-1}$, we see that $\tilde{\mathcal{L}}$ is the result of conjugation with the inverse of $\phi^{-1} \partial \phi$ :

$$
\tilde{\mathcal{L}}=\left(\phi^{-1} \partial \phi\right)^{-1} \mathcal{L}\left(\phi^{-1} \partial \phi\right)
$$

This result is compatible with the $\mathrm{KdV}$-equation in the following sense: if $q:=\partial(\phi) \cdot \phi^{-1}$ satisfies the so-called modified $\mathrm{KdV}$-equation

$$
\partial_{3}(q)=\frac{1}{4} \partial^{3}(q)-\frac{3}{2} q^{2} \partial(q),
$$

and $u$ satisfies the $\mathrm{KdV}$-equation, then also $\tilde{u}$ satisfies the $\mathrm{KdV}$-equation. This brings us in a natural way to the question we want to tackle in the present paper.

Problem. Given a solution $L$ in $R\left[\partial, \partial^{-1}\right)$ of the KP-hierarchy, determine operators $P$ and $Q$ in $R[\partial]$ such that

$$
L_{P}=P L P^{-1} \quad \text { and } \quad L_{Q}=Q^{-1} L Q
$$

are again solutions of the KP-hierarchy.

Before we can attack this problem, we first need the construction of a wide class of solutions of the KP-hierarchy besides the trivial solution $L=\partial$. These can be obtained with the help of a linear system whose compatibility leads to the equations (1.4) or (1.5). Consider namely the equations

$$
L \psi=z \psi \quad \text { and } \quad \partial_{n}(\psi)=\left(L^{n}\right)_{+}(\psi), \quad n \geq 1,
$$

where we do not specify the parameter $z$ and the eigenfuntion $\psi$ yet. If one performs on both sides of the first equation in (1.6) the following manipulations

$$
\begin{aligned}
& \partial_{n}(L \psi)=\partial_{n}(L) \psi+L \partial_{n}(\psi)=\left\{\partial_{n}(L)+L\left(L^{n}\right)_{+}\right\} \psi, \\
& \partial_{n}(z \psi)=z \partial_{n}(\psi)=z\left(L^{n}\right)_{+}(\psi)=\left(L_{+}^{n}\right)(z \psi)=\left(L_{+}^{n}\right) L \psi,
\end{aligned}
$$


then we get

$$
\left\{\partial_{n}(L)+\left[L, L_{+}^{n}\right]\right\} \psi=0 .
$$

Hence, if these manipulations make sense and if we may scratch $\psi$ in the equation (1.7), we see that the equations (1.6) imply that $L$ is a solution of the KP-hierarchy. The proper framework for these manipulations consists of the free $R\left[\partial, \partial^{-1}\right)$-module of oscillating functions.

\section{§2. Oscillating Functions}

The form of the functions on which the pseudodifferential operators from $R\left[\partial, \partial^{-1}\right)$ act, can best be understood by looking at the equations in (1.6) for the trivial solution $L=\partial$. For this solution they become

$$
\partial \psi=\frac{\partial}{\partial x} \psi=z \psi \quad \text { and } \quad \partial_{k} \psi=\frac{\partial}{\partial t_{k}} \psi=z^{k} \psi \quad \text { for all } \quad k \geq 1 .
$$

Hence the function $\gamma(x, t)=\exp \left(x z+\sum_{i \geq 1} t_{i} z^{i}\right)$ is a solution. The space $M$ of so-called oscillating functions can be seen as a collection of perturbations of this trivial solution. It is defined as

$$
M=\left\{\left(\sum_{j=-\infty}^{N} a_{j} z^{j}\right) e^{x z+\sum_{i} t_{i} z^{i}} \mid a_{i} \in R, \text { for all } i\right\},
$$

where the product $\left(\sum a_{j} z^{j}\right) e^{x z+\sum t_{i} z^{i}}$ is a formal one. The space $M$ becomes a $R\left[\partial, \partial^{-1}\right)$-module by the natural extension of the actions

$$
\begin{aligned}
& b\left\{\left(\sum_{j} a_{j} z^{j}\right) \gamma(x, t)\right\}=\left(\sum b a_{j} z^{j}\right) \gamma(x, t), \\
& \partial_{k}\left\{\left(\sum_{j} a_{j} z^{j}\right) \gamma(x, t)\right\}=\left(\sum_{j} \partial_{k}\left(a_{j}\right) z^{j}+\sum_{j} a_{j} z^{j+k}\right) \gamma(x, t), \\
& \partial\left\{\left(\sum_{j} a_{j} z^{j}\right) \gamma(x, t)\right\}=\left(\sum_{j} \partial\left(a_{j}\right) z^{j}+\sum_{j} a_{j} z^{k+1}\right) \gamma(x, t) .
\end{aligned}
$$

It is even a free $R\left[\partial, \partial^{-1}\right)$-module, since we have

$$
\left(\sum p_{j} \partial^{j}\right) \gamma(x, t)=\left(\sum p_{j} z^{j}\right) \gamma(x, t)
$$


With the space $M$ we have introduced a set-up in which the equations (1.6) make sense, where the manipulations to arrive at (1.7) are valid and that offers the possibility to leave out the function $\psi$ from equation (1.7).

Assume now that $\psi=\left(\sum_{j \leq k} \beta_{j} z^{j}\right) \gamma(x, t)=P \cdot \gamma(x, t)$ is an oscillating function with $\beta_{k} \neq 0$, for which there is a Lax operator $L$ such that the equations in (1.6) hold. Then these equations imply that $\partial\left(\beta_{k}\right)=0$ and all the $\partial_{n}\left(\beta_{k}\right)=0$. Hence, by a suitable localization, we may assume that the leading coefficient $\beta_{k}$ of $\psi$ equals 1 . An oscillating function of this form is called of type $z^{k}$. In that case the operator $P$ is invertible and the Lax operator $L$ is completely determined by $\psi$ :

$$
L=P \partial P^{-1}
$$

An oscillating function of type $z^{k}$ that satisfies the equations (1.6) for some Lax operator $L$ is called a wavefunction of the KP-hierarchy. By using the fact that $M$ is a free $R\left[\partial, \partial^{-1}\right)$-module, one proves that a Lax operator $L$ of the form (2.1) satisfies the equations (1.6) if and only if $P$ satisfies the so-called Sato-Wilson equations for $P$ :

$$
\partial_{n}(P) P^{-1}=-\left(P \partial^{n} P^{-1}\right)_{-}, \quad n \geq 1 .
$$

On $R\left[\partial, \partial^{-1}\right)$ we have a linear anti-algebra morphism called taking the adjoint. The adjoint of $P=\sum p_{i} \partial^{i}$ is given by

$$
\begin{aligned}
P^{*} & =\sum_{i}(-\partial)^{i} p_{i}=\sum_{i}(-1)^{i} \sum_{k=0}^{\infty}\left(\begin{array}{c}
i \\
k
\end{array}\right) \partial^{k}\left(p_{i}\right) \partial^{i-k} \\
& =\sum_{l}\left\{\sum_{k=0}^{\infty}(-1)^{l+k}\left(\begin{array}{c}
l+k \\
k
\end{array}\right) \partial^{k}\left(p_{l+k}\right)\right\} \partial^{l} .
\end{aligned}
$$

It is convenient to associate with this anti-involution the "adjoint" of the space $M$. This space $M^{*}$ of adjoint oscillation functions is given by all formal products

$$
\left\{\sum_{j \leq N} a_{j} z^{j}\right\} e^{-x z-\sum t_{i} z^{i}}=\left\{\sum_{j \leq N} a_{j} z^{j}\right\} \gamma(-x,-t) \text {, with } a_{j} \in R \text { for all } j .
$$

The ring $R\left[\partial, \partial^{-1}\right)$ acts as expected on $M^{*}$ by the natural extension of the actions

$$
b\left\{\left(\sum_{j} a_{j} z^{j}\right) \gamma(-x,-t)\right\}=\left(\sum_{j} b a_{j} z^{j}\right) \gamma(-x,-t)
$$




$$
\begin{gathered}
\partial_{k}\left\{\left(\sum a_{j} z^{j}\right) \gamma(-x,-t)\right\}=\left(\sum_{j} \partial\left(a_{j}\right) z^{j}-\sum_{j} a_{j} z^{k+1}\right) \gamma(-x,-t) \\
\partial\left\{\left(\sum a_{j} z^{j}\right) \gamma(-x,-t)\right\}=\left(\sum_{j} \partial\left(a_{j}\right) z^{j}-\sum_{j} a_{j} z^{1+j}\right) \gamma(-x,-t) .
\end{gathered}
$$

This is also a free $R\left[\partial, \partial^{-1}\right)$-module since we have

$$
\left(\sum_{j} p_{j}(-\partial)^{j}\right) \gamma(-x,-t)=\left(\sum p_{j} z^{j}\right) \gamma(-x,-t) .
$$

To any oscillating function $\psi=P \cdot \gamma(x, t), P \in R\left[\partial, \partial^{-1}\right)$, of type $z^{l}$ one can associate its adjoint $\psi^{*}$ in $M^{*}$ by putting

$$
\psi^{*}=\left(P^{*}\right)^{-1} \cdot \gamma(-x,-t)
$$

We call it the dual oscillating function of type $z^{-\ell}$. If $\psi \in M$ is a wavefunction for the KP-hierarchy, then its adjoint $\psi^{*}$ satisfies a similar set of linear equations, viz.,

$$
L^{*} \psi^{*}=z \psi^{*} \text { and } \partial_{n} \psi^{*}=-\left(L^{n}\right)_{+}^{*}\left(\psi^{*}\right) .
$$

There is a bilinear pairing $\mathfrak{R}: M \times M^{*} \rightarrow R$ defined as follows: if the element $\phi(x, t, z)=\left(\sum_{j} a_{j}(x, t) z^{j}\right) \gamma(x, t)$ belongs to $M$ and $\psi(x, t, z)=\left(\sum_{k} b_{k}(x, t) z^{k}\right)$ $\gamma(-x,-t)$ is an element of $M^{*}$, then we put

$$
\begin{aligned}
\mathfrak{R}(\phi, \psi): & =\operatorname{Res}_{z}\left\{\left(\sum_{j} a_{j}(x, t) z^{j}\right)\left(\sum_{k} b_{k}(x, t) z^{k}\right)\right\} \\
& =\sum_{k \in \mathbb{Z}} a_{-k-1}(x, t) b_{k}(x, t),
\end{aligned}
$$

where $\operatorname{Res}_{z} \sum_{i} a_{i} z^{i}=a_{-1}$. This is clearly a finite sum and hence belongs to $R$. There is an important connection between the bilinear form $\mathfrak{R}$ and taking the adjoint.

Proposition 2.1. Let $\phi(x, t, z)=P(x, t, \partial) \gamma(x, t) \in M$ and $\psi(x, t, z)=$ $Q(x, t, \partial) \gamma(-x,-t) \in M^{*}$, then

$$
\begin{aligned}
\left(P(x, t, \partial) Q(x, t, \partial)^{*}\right)_{-} & =\Re\left(\phi(x, t, z), \partial^{-1} \circ \psi(x, t, z)\right) \\
& :=-\sum_{n=0}^{\infty} \mathfrak{R}\left(\phi(x, t, z), \frac{\partial^{n} \psi(x, t, z)}{\partial x^{n}}\right)(-\partial)^{-n-1}
\end{aligned}
$$


Proof. The proof consists of showing, by a direct computation, that the coefficients of $\partial^{-n-1}$ on both sides of (2.4) are equal. Let $P=\sum_{j} a_{j} \partial^{j}$ and let $Q=\sum_{k} b_{k} \partial^{k}$. First we compute the coefficient $\mathfrak{R}\left(\phi(x, t, z), \partial^{n} \psi(x, t, z) /\right.$ $\left.\left(\partial x^{n}\right)\right)$. Since

$$
\begin{aligned}
\partial^{n}(\psi) & =\left\{\sum_{\ell=0}^{n}\left(\begin{array}{l}
n \\
\ell
\end{array}\right) \sum_{k} \partial^{n-\ell}\left(b_{k}\right)(-z)^{k+\ell}\right\} \gamma(-x,-t) \\
& =\left\{\sum_{p}\left(\sum_{\ell=0}^{n}\left(\begin{array}{l}
n \\
\ell
\end{array}\right)(-)^{p} \partial^{n-\ell}\left(b_{p-\ell}\right)\right) z^{p}\right\} \gamma(-x,-t),
\end{aligned}
$$

we get that

$$
\mathfrak{R}\left(\phi(x, t, z), \frac{\partial^{n} \psi(x, t, z)}{\partial x^{n}}\right)=\sum_{p} a_{-p-1} \sum_{\ell=0}^{n}\left(\begin{array}{l}
n \\
\ell
\end{array}\right)(-)^{p} \partial^{n-\ell}\left(b_{p-\ell}\right) .
$$

On the other hand we have

$$
\begin{aligned}
P Q^{*} & =\sum_{j, k} a_{j} \partial^{j}(-\partial)^{k} b_{k}=\sum_{j, k}(-)^{k} a_{j} \partial^{j+k} b_{k} \\
& =\sum_{j, k}(-)^{k} a_{j} \sum_{r=0}^{\infty}\left(\begin{array}{c}
j+k \\
r
\end{array}\right) \partial^{r}\left(b_{k}\right) \partial^{j+k-r} \\
& =\sum_{s}\left\{\sum_{j} \sum_{r=0}^{\infty}\left(\begin{array}{c}
r+s \\
r
\end{array}\right)(-)^{r+s+j} a_{j} \partial^{r}\left(b_{r+s-j}\right)\right\} \partial^{s} .
\end{aligned}
$$

Hence the coefficient of $\partial^{-n-1}, n \geq 0$, equals

$$
\begin{aligned}
(-)^{n+1} \sum_{j} \sum_{r=0}^{\infty}\left(\begin{array}{c}
r-n-1 \\
r
\end{array}\right) & (-)^{r+j} a_{j} \partial^{r}\left(b_{r-j-n-1}\right) \\
& =(-)^{n+1} \sum_{j} \sum_{r=0}^{n}\left(\begin{array}{c}
n \\
r
\end{array}\right)(-)^{j} a_{j} \partial^{r}\left(b_{r-j-n-1}\right) \\
& =(-)^{n+1} \sum_{j}(-)^{j} a_{j} \sum_{\ell=0}^{n}\left(\begin{array}{l}
n \\
\ell
\end{array}\right) \partial^{n-\ell}\left(b_{-\ell-j-1}\right) \\
& =(-)^{n} \sum_{p}(-)^{p} a_{-p-1} \sum_{\ell=0}^{n}\left(\begin{array}{l}
n \\
\ell
\end{array}\right) \partial^{n-\ell}\left(b_{p-\ell}\right) \\
& =(-)^{n} \Re\left(\phi(x, t, z), \frac{\partial^{n} \psi(x, t, z)}{\partial x^{n}}\right) .
\end{aligned}
$$


This proves the claim in the proposition.

This shows that the adjoint $\phi^{*}$ of an oscillating function $\phi \in M$ of type $z^{\ell}$ satisfies for all $n \geq 0$

$$
\mathfrak{R}\left(\phi(x, t, z), \frac{\partial^{n} \phi^{*}(x, t, z)}{\partial x^{n}}\right)=0 \quad \text { for all } n \geq 0 .
$$

This property even characterizes $\phi^{*}$ among the dual oscillating functions of type $z^{-\ell}$. For, if $\psi=Q(x, t, \partial) \gamma(-x,-t) \in M^{*}$ is such a function satisfying (2.5) with $\phi^{*}$ replaced by $\psi$, then we have according to the theorem

$$
P Q^{*}=\partial^{0}+\left(P Q^{*}\right)_{-}=1 .
$$

In other words, $Q=\left(P^{*}\right)^{-1}$ and $\psi=\phi^{*}$. We will use this criterion later on and therefore we resume it in a

Lemma 2.1. Let $\phi$ be an oscillating function of type $z^{\ell}$ and $\psi$ a dual oscillating function of type $z^{-\ell}$. Then $\psi$ is the adjoint of $\phi$ if and only if it satisfies

$$
\mathfrak{R}\left(\phi(x, t, z), \frac{\partial^{n} \psi(x, t, z)}{\partial x^{n}}\right)=0 \text { for all } n \geq 0 .
$$

The bilinear form $\mathfrak{R}$ plays also an important role at the interpretation of the famous bilinear identities for an oscillating function $\psi$ and its dual, (see $[9])$ :

$$
\operatorname{Res}_{z} \psi(x, t, z) \psi^{*}(y, s, z)=0 .
$$

For, if $e^{x z+\sum t_{i} z^{i}}=\sum_{r=0}^{\infty} p_{r}\left(x,\left(t_{i}\right)\right) z^{r}$ and we have $\psi(x, t, z)=\left\{\sum_{j \leq 0}\right.$ $\left.a_{j}(x, t) z^{j}\right\} z^{l} \gamma(x, t)$ and $\psi^{*}(x, s, z)=\left\{\sum_{m \leq 0} b_{m}(x, s) z^{m}\right\} z^{-l} \gamma(-x,-t)$, then this equation boils formally down to

$$
\left\{\sum_{r=0}^{\infty} p_{r}\left(x,\left(t_{i}-s_{i}\right)\right)\left\{\sum_{j=-r-1}^{0} a_{j}(x, t) b_{-r-1-j}(x, s)\right\}\right\}=0,
$$

for all relevant $t=\left(t_{i}\right)$ and $s=\left(s_{i}\right)$. In order to avoid convergence considerations, we look at a few differential consequences of these relations that always exist. If one differentiates the equation namely with respect to some of the parameters $\left(t_{i}\right)$ or $\left(s_{i}\right)$ and substitutes next $t_{i}=s_{i}$ for all $i \geq 1$, then one ends up with finite expressions in the coefficients of $\psi$ and $\psi^{*}$. Thus we get for example for all $k \geq 0$, all $n \geq 1$ and all $m \geq 0$ that

$$
\mathfrak{R}\left(\partial^{k}(\psi(x, t, z)), \partial^{m}\left(\psi^{*}(x, t, z)\right)\right)=0 \text { and }
$$




$$
\mathfrak{R}\left(\partial_{n}(\psi(x, t, z)), \partial^{m}\left(\psi^{*}(x, t, z)\right)\right)=0 .
$$

Note that this first equation can also be obtained by applying $\partial$ several times to relations (2.5). Assume now that an oscillating function $\psi$ of type $z^{l}$ and its dual $\psi^{*}$ satisfy the equations $(2.7)$ and let $L=P \partial P^{-1}$ be the Lax operator associated with $\psi$. From the form of the action of the $\partial_{n}$ on $\psi$ one sees that there exists for each $n \geq 1$ a monic differential operator $Q_{n}$ in $R[\partial]$ of degree $n$ such that

$$
\begin{aligned}
\partial_{n}(\psi)-Q_{n}(\psi) & =G_{n} \partial^{l} \gamma(x, t) e^{x z+\sum_{i} t_{i} z^{i}}, \text { with } \\
G_{n} & \in R\left[\partial, \partial^{-1}\right) \text { of degree }<0 .
\end{aligned}
$$

Because of the relations in (2.7) we have then for all $m \geq 0$ that

$$
\mathfrak{R}\left(\partial_{n}(\psi(x, t, z))-\left(Q_{n}\right)(\psi(x, t, z)), \partial^{m}\left(\psi^{*}(x, t, z)\right)\right)=0 .
$$

According to Proposition 2.1, we get then that $\left(G_{n} \partial^{l} P^{-1}\right)_{-}=G_{n} \partial^{l} P^{-1}=0$. Thus we have obtained that $\partial_{n}(\psi(x, t, z))-\left(Q_{n}\right)(\psi(x, t, z))=0$. It is wellknown that this implies for all $n \geq 1$ that the operator $Q_{n}$ is equal to $\left(L^{n}\right)_{+}$. Hence $\psi$ is a wavefunction of the KP-hierarchy. Reversely, if $\psi$ is a wavefunction of the KP-hierarchy, then the first equation in (2.7) holds and since $\partial_{n}$ acts on $\psi$ as $\left(L^{n}\right)_{+}$also the second relation holds. Thus we have found the following characterization of the wavefunctions in $M$.

Proposition 2.2. $\quad$ An oscillating function $\psi$ of type $z^{l}$ is a wavefunction of the KP-hierarchy if and only if $\psi$ and its dual $\psi^{*}$ satisfy the equations (2.7).

A consequence of this proposition is that wavefunctions of the KPhierarchy satisfy the following set of equations

$$
\mathfrak{R}\left(\Delta_{1}(\psi(x, t, z)), \Delta_{2}\left(\psi^{*}(x, t, z)\right)\right)=0,
$$

where $\Delta_{1}$ and $\Delta_{2}$ are arbitrary finite products of the $\left\{\partial_{n}\right\}$ and $\partial$. These equations also characterize the wavefunctions of the KP-hierarchy and are the algebraic version of the equations (2.6).

\section{$\S 3 . \quad$ The Sato Grassmannian}

In this section we describe the Sato Grassmannian, from which one can construct wavefunctions of the KP-hierarchy. Since these functions involve 
infinite series in $z$ and $z^{-1}$, it is not so strange that the basic manifold for the relevant subspaces consists of a class of series in $z$ and $z^{-1}$. Consider the spaces

$$
\begin{aligned}
& H_{-}=z^{-1} \mathbb{C}\left[\left[z^{-1}\right]\right]=\left\{\sum_{j=1}^{\infty} a_{j} z^{-j} \mid a_{j} \in \mathbb{C}\right\} \text { and } \\
& H_{+}=\mathbb{C}[z]=\left\{\sum_{i=0}^{m} b_{i} z^{i} \mid b_{i} \in \mathbb{C}\right\} .
\end{aligned}
$$

Hence $H=H_{+} \oplus H_{-}$is the quotient field $\mathbb{C}((z))$ of $\mathbb{C}\left[\left[z^{-1}\right]\right]$. Let $p_{+}: H \rightarrow H_{+}$ be the projection

$$
p_{+}\left(\sum a_{j} z^{j}\right)=\sum_{j \geq 0} a_{j} z^{j} .
$$

Then the Grassmann manifold of Sato consists of all subspaces of $H$ that are of a size comparable to $H_{+}$. More precisely, it is given by

$$
G r(H)=\left\{\begin{array}{l|l}
W & \begin{array}{l}
W \subset H, p_{+}: W \rightarrow H_{+} \text {has a finite } \\
\text { dimensional kernel and cokernel }
\end{array}
\end{array}\right\} .
$$

The space $G r(H)$ has a subdivision into different components according to the index of $\left.p_{+}\right|_{W}$ that is defined by

$$
\text { ind }\left(\left.p_{+}\right|_{W}\right)=\operatorname{dim}\left(\operatorname{Ker}\left(\left.p_{+}\right|_{W}\right)\right)-\operatorname{dim}\left(\operatorname{Coker}\left(\left.p_{+}\right|_{W}\right)\right) .
$$

We denote these components as follows

$$
G r^{(k)}(H)=\left\{W \mid W \in G r(H), \operatorname{ind}\left(\left.p_{+}\right|_{W}\right)=-k\right\} .
$$

Clearly, the subspace $z^{k} H_{+}$belongs to $G r^{(k)}(H)$ and one easily verifies that this also holds for all subspaces in $G r(H)$ that project bijectively onto $z^{k} H_{+}$, i.e. all $W$ belonging to the "big cell"

$$
O^{(k)}=\left\{\begin{array}{l|l}
W & \begin{array}{l}
W \in G r(H), w \mapsto z^{k} p_{+}\left(z^{-k} w\right) \\
\text { bijection: } W \rightarrow z^{k} H_{+}
\end{array}
\end{array}\right\} .
$$

To have a description of all planes in $G r^{(k)}(H)$, consider for each $k$ in $\mathbb{Z}$ the collection of sequences

$$
\mathcal{S}(k)=\left\{\left(s_{i}\right) \begin{array}{l}
i \in \mathbb{Z}, i \geq k, s_{i} \in \mathbb{Z}, s_{i+1}>s_{i} \text { and } s_{l}=l \\
\text { for } l \text { sufficiently large }
\end{array}\right\} .
$$


For $\sigma=\left(s_{i}\right)$ in $\mathcal{S}(k)$, one has the subspace $H(\sigma)$ in $G r^{(k)}(H)$ given by

$$
H(\sigma)=\operatorname{Span}\left\{z^{s_{i}} \mid \sigma=\left(s_{i}\right)\right\}
$$

Let $p(\sigma): H \rightarrow H(\sigma)$ be the projection

$$
p(\sigma)\left(\sum \alpha_{i} z^{i}\right)=\sum_{s_{i}, \sigma=\left(s_{i}\right)} \alpha_{s_{i}} z^{s_{i}}
$$

Since each plane in $G r(H)$ has a basis of elements with different leading coefficients, one sees that there exists for each $W \in G r^{(k)}(H)$ a $\sigma$ in $\mathcal{S}(k)$ such that the projection $p(\sigma): W \rightarrow H(\sigma)$ is a bijection. As each $H(\sigma), \sigma=\left(s_{j}\right) \in \mathcal{S}(k)$, is the image of the embedding $i_{\sigma}: z^{k} H_{+} \rightarrow H$ given by

$$
i_{\sigma}\left(z^{j}\right)=z^{s_{j}}, \quad j \geq k
$$

one sees that the planes in $G r^{(k)}(H)$ can be described as follows

Proposition 3.1. Each $W \in G r^{(k)}(H)$ is the image of an embedding $w: z^{k} H_{+} \rightarrow H$ such that, if $w\left(z^{j}\right)=\sum_{i} w_{i j} z^{i}$ for all $j \geq k$, the upper part $w_{+}$ of the matrix $[w]=\left(w_{i j}\right)$ has the form

$$
w_{+}=\left(\begin{array}{ccc}
\ddots & & \vdots \\
& w_{k+1, k+1} & w_{k+1, k} \\
\ldots & w_{k, k+1} & w_{k, k}
\end{array}\right)=\left(\begin{array}{cc}
I d & 0 \\
B & A
\end{array}\right),
$$

where $A$ is a square matrix of finite size. Reversely, for every such embedding $w$, its image belongs to $G r^{(k)}(H)$. In particular $w\left(z^{k} H_{+}\right)$belongs to the big cell iff $\operatorname{det}(A)$ is non zero.

Notation. We denote the collection of embeddings $w: z^{k} H_{+} \rightarrow H$ that occur in the proposition by $\mathcal{P}_{k}$.

On the space $H$ we have a bilinear form $B$ that plays a role in the sequel. Namely, if $f=\sum_{j} a_{j} z^{j}$ and $g=\sum_{j} b_{j} z^{j}$ are in $H$, then we define

$$
B(f, g)=\operatorname{Res}_{z}(f(z) g(z))=\sum_{j} a_{j} b_{-j-1} .
$$

For $W$ in $\operatorname{Gr}(H)$, let $W^{\perp}$ be the orthocomplement of $W$ in $H$ w.r.t. this form $B$. With the above given description of spaces in $G r(H)$ one verifies that $W^{\perp}$ also belongs to $G r(H)$.

Our next step will be to show how the subspaces from $\operatorname{Gr}(H)$ occur as the span of the Laurent coefficients of certain oscillating functions. First we 
describe the ring $R$ and its derivations $\partial_{n}$ and $\partial$. Consider the ring $\mathbb{C}[[x, t]]$ of formal powerseries in $x$ and $t=\left(t_{i}\right)$. Let $\alpha$ be an element in $\mathbb{C}[[x, t]]$ of the form

$$
\alpha(x, 0)=x^{N}+\sum_{j>N} a_{j} x^{j}
$$

In the sequel the ring $R$ will always be the localization of $\mathbb{C}[[x, t]]$ w.r.t. $S_{\alpha}=$ $\left\{\alpha^{m} \mid m \geq 0\right\}$ for some $\alpha$ of the form (3.2). On $R$ we take $\partial_{n}=\partial /\left(\partial t_{n}\right)$ and $\partial=\partial /(\partial x)$. Consider a wavefunction $\psi$ of the KP-hierarchy and its dual $\psi^{*}$ that have the form

$$
\begin{aligned}
\psi(x, t, z) & =\left\{\sum_{j \leq 0} a_{j}(x, t) z^{j}\right\} z^{l} \gamma(x, t) \text { and } \\
\psi^{*}(x, t, z) & =\left\{\sum_{m \leq 0} b_{m}(x, t) z^{m}\right\} z^{-l} \gamma(-x,-t) .
\end{aligned}
$$

The class of wavefunctions we will consider in this paper that satisfies the condition that there is an $\alpha$ of the form (3.2) such that for all $m \leq 0$ and all $j \leq 0$

$$
\alpha(x, t) a_{j}(x, t) \in \mathbb{C}[[x, t]] \text { and } \alpha(x, t) b_{m}(x, t) \in \mathbb{C}[[x, t]] .
$$

These wavefunctions are called regularizable. For regularizable wavefunctions the Laurent series in $x$ of $\psi$ and $\psi^{*}$ have the form

$$
\begin{aligned}
\psi(x, t, z) & =\left\{\sum_{j \geq-N} w_{j}(t, z) x^{j}\right\} \gamma(x, t), \text { where } w_{j}(t, z) \\
& =\sum_{l=-\infty}^{N_{1}} v_{l} z^{l}, \text { with } v_{l} \in \mathbb{C}[[t]], \\
\psi^{*}(x, t, z) & =\left\{\sum_{j \geq-N} w_{j}^{*}(t, z) x^{j}\right\} \gamma(-x,-t), \text { where } w_{j}^{*}(t, z) \\
& =\sum_{l=-\infty}^{N_{2}} v_{l}^{*} z^{l}, \text { with } v_{l}^{*} \in \mathbb{C}[[t]] .
\end{aligned}
$$

It is not hard to show that both spaces

$$
W=\operatorname{Span}\left\{w_{j}(0, z), j \geq-N\right\} \text { and } W^{*}=\operatorname{Span}\left\{w_{j}^{*}(0, z), j \geq-N\right\}
$$


belong to $\operatorname{Gr}(H)$. It was Sato who realized that the space $W$ even determines $\psi$, for according to $([24])$ there holds

Proposition 3.2. $\quad$ The map that associates to a regularizable wavefunction $\psi$ of the KP-hierarchy the span of the coefficients in $t=0$ of the Laurent series of $\psi$ in $x$ is a bijection between this class of wavefunctions and $G r(H)$. The wavefunctions that satisfy the conditions in (3.3) for $\alpha=1$ correspond to the union of the big cells. For each $W \in G r(H)$ we denote the wavefunction corresponding to $W$ by $\psi_{W}$.

Now that we have this link between wavefunctions and the Grassmann manifold, we can give a geometric description of the dual wavefunction of $\psi_{W}$, which we denote by $\psi_{W}^{*}$. From the characterizing properties in (2.7), follows

Proposition 3.3. $\quad$ Let $W$ and $\tilde{W}$ be two subpaces in $\operatorname{Gr}(H)$. Then $\tilde{W}$ is the space $W^{*}$ corresponding to the dual wavefunction, if and only if $\tilde{W}=W^{\perp}$ with $W^{\perp}$ the orthocomplement of $W$ w.r.t. the bilinear form $B$ on $H$.

\section{$\S 4$. The Tau-Functions}

In the foregoing section we saw how one could associate to a regularizable wavefunction of the KP-hierarchy a plane in $\operatorname{Gr}(H)$ and that the class of wavefunctions of the KP-hierarchy is in bijection with $G r(H)$. The present section is devoted to an explicit description of the inverse of this map, i.e. we will show how to build such a wavefunction, starting from an element in the Grassmann manifold. Let $W \in G r^{(k)}(H)$ be the image of an embedding $w: z^{k} H_{+} \rightarrow H$ in $\mathcal{P}_{k}$. If $w\left(z^{j}\right)=\sum_{i \in \mathbb{Z}} w_{i j} z^{i}$ for all $j \geq k$, then the matrix of $w$ decomposes w.r.t. $H=z^{k} H_{+} \oplus z^{k} H_{-}$in the components $w_{+}=\left(w_{i j}\right), i \geq k$ and $j \geq k$ and $w_{-}=\left(w_{i j}\right), i \geq k$ and $j<k$. Since $w$ belongs to $\mathcal{P}_{k}$, we may assume that the $\mathbb{Z}_{\geq k} x \mathbb{Z}_{\geq k}$-matrix $w_{+}$has w.r.t. $z^{k} H_{+}=z^{l} H_{+} \oplus \operatorname{Span}\left(z^{m}, \quad k \leq m<l\right)$ for sufficiently large $l$ the form

$$
w_{+}=\left(\begin{array}{cc}
I d & 0 \\
B & A
\end{array}\right) .
$$

Though the multiplication of elements in $H$ with the exponential factor $e^{x z+\sum_{i} t_{i} z^{i}}$ brings them outside of $H$, we will nevertheless consider the matrix with respect to this operation. First we write

$$
\gamma(x, t)=e^{x z+\sum_{i} t_{i} z^{i}}=\sum_{i=0}^{\infty} p_{l}(x, t) z^{l},
$$


where $p_{l}$ a homogeneous polynomial in $\mathbb{C}[[x, t]]$ of degree 1 , if we put the degree of $x$ equal to one and that of $t_{n}$ equal to $\mathrm{n}$. Then the corresponding $\mathbb{Z} \times \mathbb{Z}$-matrix $[\gamma]=\left(\gamma_{i j}\right)$ with coefficients in $\mathbb{C}[[x, t]]$ is given by

$$
\gamma_{i j}=0, \text { if } i<j \text { and } \gamma_{i j}=p_{i-j} \text { for } i \geq j .
$$

Also the matrix $[\gamma]$ we decompose w.r.t. $H=z^{k} H_{+} \oplus z^{k} H_{-}$as

$$
[\gamma]=\left(\begin{array}{cc}
\alpha & \beta \\
0 & \delta
\end{array}\right)
$$

Clearly, the $\mathbb{Z}_{\geq k} \times \mathbb{Z}_{\geq k}$-matrix $\alpha$ is invertible within the $\mathbb{Z}_{\geq k} \times \mathbb{Z}_{\geq k}$-matrices with coefficients from $\mathbb{C}[[x, t]]$. One verifies directly that the product $[\gamma][w] \alpha^{-1}$ is a well-defined $\mathbb{Z} \times \mathbb{Z}_{\geq k}$-matrix with coefficients in $\mathbb{C}[[x, t]]$. From the special form of $w_{+}$one sees directly that the $\mathbb{Z}_{\geq k} \times \mathbb{Z}_{\geq k}$-matrix

$$
\left([\gamma][w] \alpha^{-1}\right)_{+}=\alpha w_{+} \alpha^{-1}+\beta w_{-} \alpha^{-1}
$$

is the sum of an invertible $\mathbb{Z}_{\geq k} \times \mathbb{Z}_{\geq k}$-matrix with coefficients from $\mathbb{C}[[x, t]]$ and one with a finite dimensional range, hence it has a well-defined determinant belonging to $\mathbb{C}[[x, t]]$. We define now the $\tau$-function corresponding to $w$ by

$$
\tau_{w}(x, t):=\operatorname{det}\left(\alpha w_{+} \alpha^{-1}+\beta w_{-} \alpha^{-1}\right) .
$$

By using the fact that there exists for $W$ a $H(\sigma)$ with $\sigma$ in $\mathcal{S}(k)$ such that $W$ projects bijectively onto $H(\sigma)$, one derives that $\tau_{w}$ satisfies condition (3.2). The following key result now is due to Sato, see e.g. ([18])

Proposition 4.1. Let $w \in \mathcal{P}_{k}$ be an embedding of $z^{k} H_{+}$into $H$ with image $W$. Then the following formulae define a regularizable wavefunction of the KP-hierarchy of type $z^{k}$ and its dual, respectively,

$$
\begin{aligned}
& \psi_{W}(x, t, z)=\frac{\tau_{w}\left(x,\left(t_{i}-\frac{1}{i z^{i}}\right)\right)}{\tau_{w}(x, t)} z^{k} \gamma(x, t), \\
& \psi_{W}^{*}(x, t, z)=\frac{\tau_{w}\left(x,\left(t_{i}+\frac{1}{i z^{i}}\right)\right)}{\tau_{w}(x, t)} z^{-k} \gamma(-x,-t) .
\end{aligned}
$$

Now multiply the $\psi_{W}$ of the proposition with $z^{-k} e^{x z+\sum_{i} t_{i} z^{i}}$ and take derivatives with respect to $z$ and all times $t_{j}$, then one deduces that

$$
\partial_{\ell} \log \left(\tau_{w}(x, t)\right)=\operatorname{Res}_{z}\left(\left(\frac{\partial}{\partial z}-\sum_{j=1}^{\ell-1} z^{-j-1} \partial_{j}\right) \log \left(z^{-k} \gamma(x, t) \psi_{W}(x, t, z)\right)\right) .
$$


From this formula we see that if there are two such tau-functions that give the same wavefunction then their quotient is independent of the variables $\left(t_{\ell}\right)$. For this reason we will write from now on $\tau_{W}$ instead of $\tau_{w}$.

Let $\tau$ be an element of $\mathbb{C}[[x, t]]$ of the form (3.2). Then we can define an oscillating function $\phi_{\tau}$ of type $z^{k}$ by the formula

$$
\phi_{\tau}(x, t, z)=\frac{\tau\left(x,\left(t_{i}-\frac{1}{i z^{i}}\right)\right)}{\tau(x, t)} z^{k} e^{x z+\sum_{i} t_{i} z^{i}},
$$

As we have shown in Theorem (2.2) this $\phi_{\tau}$ is a wavefunction of the KPhierarchy if and only if it satisfies the equations (2.7). By expressing these equations in $\tau$, one ends up with a collection of so-called Hirota bilinear identities for $\tau$, i.e. equations of the form

$$
P\left(\left(D_{i}\right)\right) \tau . \tau:=\left.P\left(\left(D_{i}\right)\right)\left(\tau\left(x,\left(t_{i}-y_{i}\right)\right) \tau\left(x,\left(t_{i}+y_{i}\right)\right)\right)\right|_{\left(y_{i}\right)=0}=0,
$$

where $D_{i}=\partial /\left(\partial y_{i}\right)$ and $P$ is some polynomial in these derivatives. Like the characterizing properties for wavefunctions are symbolically written in the form (2.6), we have a similar form for the bilinear identity that characterizes the KP tau-functions:

$$
\operatorname{Res}_{z} \tau\left(x, t-\left[z^{-1}\right]\right) e^{\sum\left(t_{i}-s_{i}\right) z^{i}} \tau\left(y, s+\left[z^{-1}\right]\right)=0,
$$

where $[u]=\left(u, u^{2} / 2, u^{3} / 3, \ldots\right)$. As a consequence of the Sato-Wilson equations (2.2) one finds that the $x$ and $t_{1}$ dependence of the wavefunction is as follows:

$$
\psi_{W}\left(x, t_{1}, t_{2}, t_{3}, \ldots, z\right)=\psi_{W}\left(0, x+t_{1}, t_{2}, t_{3}, \ldots, z\right)
$$

and similarly for the adjoint wavefunction and tau-function. To avoid lengthy notations, we will identify from now on $x$ with $t_{1}$ and write $\psi(t, z), \psi^{*}(t, z)$ and $\tau(t, z)$. We will use both $\partial /(\partial x)$ and $\partial /\left(\partial t_{1}\right)$ for $\partial /\left(\partial t_{1}\right)$.

\section{§5. Main Theorem}

We merely need one more ingredient to state the Main Theorem. Let

$$
\left\langle\sum_{i} a_{i} z^{i} \mid \sum_{j} b_{j} z^{j}\right\rangle:=\operatorname{Res}_{z} \sum_{i, j} a_{i} b_{j} z^{i+j}=\sum_{i} a_{i} b_{-i-1},
$$

then one has 
Theorem 5.1. Let $V \in G r^{(k)}$ and $W \in G r^{(\ell)}$ be such that $V \cap W$ has codimension $m$ in $V$ and codimension $n$ in $W$ (hence $k-m=\ell-n)$ and let $\psi_{W}$, $\psi_{W}^{*}$ and $\tau_{W}$ be the corresponding wave-, adjoint wave- and tau-function. Then the corresponding Bäcklund-Darboux transformation $B D(V, W)$ that maps $\psi_{W}$ into $\psi_{V}$ is equal to

$$
\begin{aligned}
B D(V, W)= & \mathcal{W}\left(\psi_{W} ; w_{1}, \ldots, w_{n} ; \psi_{W}^{*} ; v_{1}, \ldots, v_{m}\right)^{-1} \\
& \times \mathcal{W}\left(\psi_{W} ; w_{1}, \ldots, w_{n} ; \psi_{W}^{*} ; v_{1}, \ldots, v_{m} ; \partial\right)
\end{aligned}
$$

where

$$
\begin{aligned}
& \mathcal{W}\left(A ; w_{1}, \ldots, w_{n} ; B ; v_{1}, \ldots, v_{m}\right)=
\end{aligned}
$$

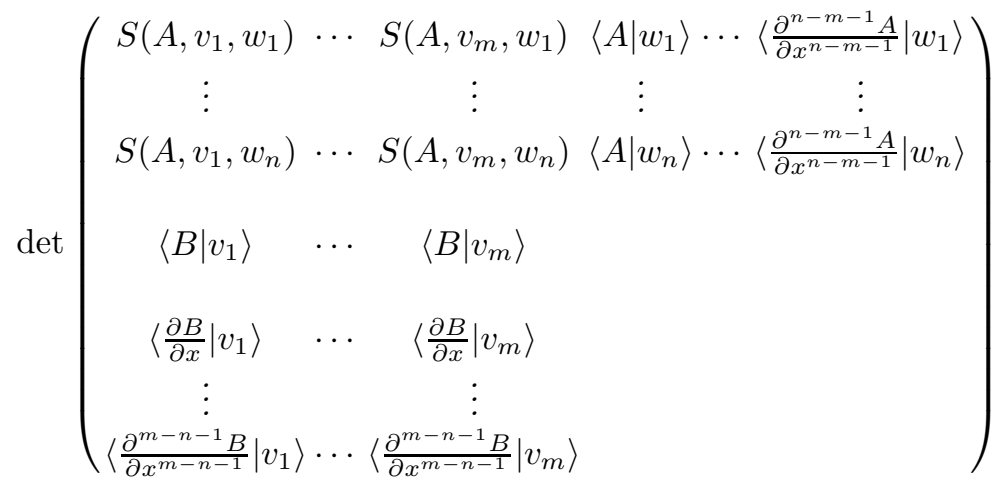

and

(5.3) $\mathcal{W}\left(A ; w_{1}, \ldots, w_{n} ; B ; v_{1}, \ldots, v_{m} ; \partial\right)=$

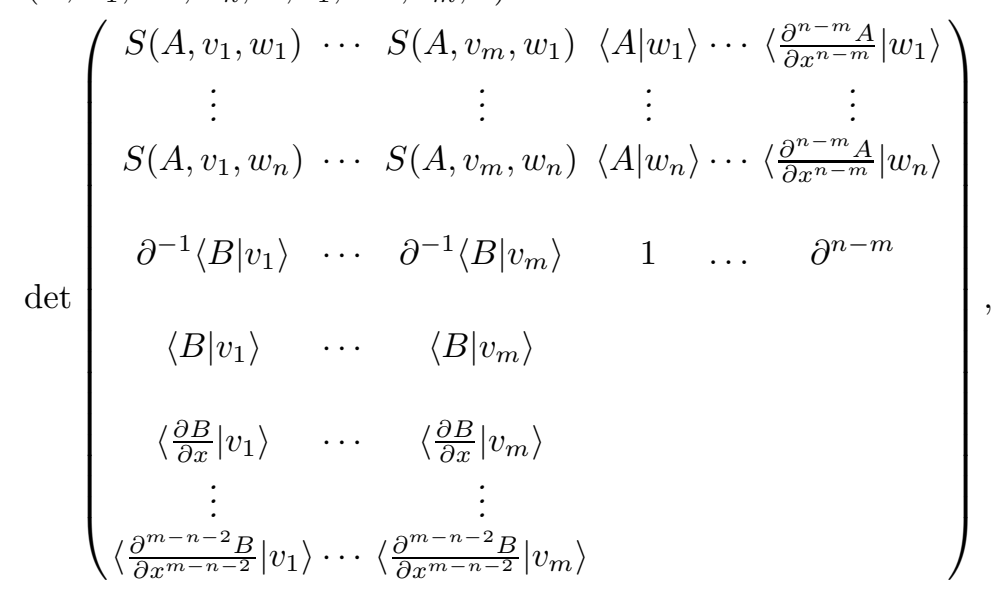


and its adjoint inverse is equal to

$$
\begin{aligned}
B D(V, W)^{*-1}= & (-)^{n+m} \mathcal{W}\left(\psi_{W}^{*} ; v_{1}, \ldots, v_{m} ; \psi_{W} ; w_{1}, \ldots, w_{n}\right)^{-1} \\
& \times \mathcal{W}\left(\psi_{W}^{*} ; v_{1}, \ldots, v_{m} ; \psi_{W} ; w_{1}, \ldots, w_{n} ; \partial\right)
\end{aligned}
$$

Here we have taken elements $w_{1}, w_{2}, \ldots, w_{n}$ in $V^{\perp}$ such that

$$
V=\left\{x \mid x \in V+W,\left\langle x \mid w_{i}\right\rangle=0 \text { for all } i\right\},
$$

and elements $v_{1}, v_{2}, \ldots, v_{m}$ in $V+W$ such that

$$
(V+W)^{\perp}=\left\{x \mid x \in W^{\perp},\left\langle x \mid v_{j}\right\rangle=0 \text { for all } j\right\} .
$$

Moreover,

$$
\begin{aligned}
\psi_{V}= & \mathcal{W}\left(\psi_{W} ; w_{1}, \ldots, w_{n} ; \psi_{W}^{*} ; v_{1}, \ldots, v_{m}\right)^{-1} \\
& \times \mathcal{W}\left(\psi_{W} ; w_{1}, \ldots, w_{n} ; \psi_{W}^{*} ; v_{1}, \ldots, v_{m} ; \psi_{W}\right), \\
\psi_{V}^{*}= & (-)^{n+m} \mathcal{W}\left(\psi_{W}^{*} ; v_{1}, \ldots, v_{m} ; \psi_{W} ; w_{1}, \ldots, w_{n}\right)^{-1} \\
& \times \mathcal{W}\left(\psi_{W}^{*} ; v_{1}, \ldots, v_{m} ; \psi_{W} ; w_{1}, \ldots, w_{n} ; \psi_{W}^{*}\right),
\end{aligned}
$$

and

$$
\tau_{V}=\mathcal{W}\left(\psi_{W} ; w_{1}, \ldots, w_{n} ; \psi_{W}^{*} ; v_{1}, \ldots, v_{m}\right) \tau_{W}
$$

where $\mathcal{W}\left(A ; w_{1}, \ldots, w_{n} ; B ; v_{1}, \ldots, v_{m} ; C\right)$ is the determinant of the same matrix as the one appearing in (5.3), except that one has to replace the $(n+1)$-th row by

$$
\left(S\left(C, v_{1}\right), \ldots, S\left(C, v_{m}\right), C, \ldots, \frac{\partial^{n-m} C}{\partial x^{n-m}}\right)
$$

where $S(C, v)=\partial^{-1}(\langle B \mid v\rangle C)$ is defined in (7.2) and $S(A, v, w)=\langle S(A, v) \mid w\rangle$ (see $(8.3))$.

The matrix appearing in (5.2) is an $n \times n$-matrix if $n \geq m$ and an $m \times m$ matrix if $n<m$. The matrix appearing in (5.3) is an $n+1 \times n+1$-matrix if $n \geq m$ and an $m \times m$-matrix if $n<m$. The determinant appearing in (5.3) is the formal expansion with respect to the $(n+1)$-th row putting all the minors to the left of the $\partial$ symbols. The theorem generalizes a result of Crumm [8]. The proof of this theorem will be given in Section 8. We first need a simpler version of this theorem, which we will give and proof in the next section. 


\section{§6. Trivial and Elementary Bäcklund-Darboux Transformations}

In Section 8 we want to proof the main theorem of this paper. But before doing this we discuss trivial and elementary Bäcklund-Darboux transformations. The latter will form the building blocks of the more complicated ones that appear in the main theorem. For some literature on Bäcklund-Darboux transformations we refer the reader to [2], [5], [17], [19] and [20].

Let $L=L(t, z)$ be the Lax operator satisfying (1.4), and suppose that $\psi_{j}(t, z)=P_{j}(t, \partial) e^{\sum_{i} t_{i} z^{i}}, j=1,2$, are wavefunction of type $z^{\ell_{j}}$ corresponding to points of the Grassmannian $W_{j}$, such that $L=P_{j} \partial P_{j}^{-1}$ for both $j=1,2$. Then $\partial\left(P_{2}^{-1} P_{1}\right)=0$ and using this and the Sato-Wilson equations $(2.2)$ one deduces that also $\left(\partial\left(P_{2}^{-1} P_{1}\right)\right) /\left(\partial t_{k}\right)=0$. Hence $P_{2}^{-1} P_{1}=\partial^{\ell_{1}-\ell_{2}}+\sum_{k<\ell_{1}-\ell_{2}} a_{k} \partial^{k}$ is an invertible pseudo-differential operator with constant coefficients. Therefore, $W_{1}=R(z) W_{2}$ with

$$
\begin{aligned}
R(z) & =z^{\ell_{1}-\ell_{2}}+\sum_{k<\ell_{1}-\ell_{2}} a_{k} z^{k} \text { all } a_{k} \in \mathbb{C} \text { and } \\
\psi_{1}(t, z) & =R(z) \psi_{2}(t, z)=P_{2}(t, \partial) R(\partial) e^{\sum_{i} t_{i} z^{i}} .
\end{aligned}
$$

These kind of transformations which map $\psi_{2}$ into $\psi_{1}$ are called trivial Bäcklund-Darboux transformations, because they do not change the Lax operator $L$. However, they do change the tau-function. Suppose $\psi_{2}$ is a wavefunction with tau-function $\tau_{2}$ and let $R(z)$ be as in (6.1) with $k=\ell_{1}-\ell_{2}$. Then clearly since all coefficients of $R(z)$ are constants, $\psi_{1}(t, z)=R(z) \psi_{2}(t, z)$ is again a wavefunction. The corresponding adjoint wavefunction is given by

$$
\psi_{1}^{*}(t, z)=P_{2}(t, \partial)^{*-1} R(\partial)^{*-1} e^{-\sum_{i} t_{i} z^{i}}=R(z)^{-1} \psi_{2}^{*}(t, z) .
$$

Rewrite $R(z)$ as follows

$$
R(z)=z^{k} e^{-\sum_{j=1}^{\infty} \frac{q_{j}}{j} z^{-j}}
$$

then $\psi_{1}(t, z)=R(z) \psi_{2}(z)$

$$
\begin{aligned}
\psi_{1}(t, z) & =z^{k} e^{-\sum_{j=1}^{\infty} \frac{q_{j}}{j} z^{-j}} \psi_{2}(t, z) \\
& =z^{k+\ell} \frac{\tau_{2}\left(t-\left[z^{-1}\right]\right)}{\tau_{2}(t)} e^{-\sum_{j=1}^{\infty} \frac{q_{j}}{j} z^{-j}} e^{\sum_{i=1}^{\infty} t_{i} z^{i}} \\
& =z^{k+\ell} \frac{\tau_{2}\left(t-\left[z^{-1}\right]\right) e^{\sum_{j=1}^{\infty} q_{j}\left(t_{j}-\frac{z^{-j}}{j}\right)}}{\tau_{2}(t) e^{\sum_{j=1}^{\infty} q_{j} t_{j}}} e^{\sum_{i=1}^{\infty} t_{i} z^{i}},
\end{aligned}
$$


which suggests that $\tau_{1}(t):=\tau_{2}(t) e^{\sum_{j=1}^{\infty} q_{j} t_{j}}$ is the to $\psi_{1}$ corresponding taufunction. Since

$$
\psi_{1}^{*}(t, z)=z^{-k-\ell} \frac{\tau_{2}\left(t+\left[z^{-1}\right]\right) e^{\sum_{j=1}^{\infty} q_{j}\left(t_{j}+\frac{z^{-j}}{j}\right)}}{\tau_{2}(t) e^{\sum_{j=1}^{\infty} q_{j} t_{j}}} e^{-\sum_{i=1}^{\infty} t_{i} z^{i}},
$$

one deduces the following bilinear identity for $\tau_{1}$ :

$$
\begin{gathered}
\operatorname{Res}_{z} \tau_{2}\left(t-\left[z^{-1}\right]\right) e^{\sum_{j=1}^{\infty} q_{j}\left(t_{j}-\frac{z^{-j}}{j}\right)} e^{\sum_{i=1}^{\infty}\left(t_{i}-s_{i}\right) z^{i}} \\
\cdot \tau_{2}\left(s+\left[z^{-1}\right]\right) e^{\sum_{j=1}^{\infty} q_{j}\left(s_{j}+\frac{z^{-j}}{j}\right)}=0
\end{gathered}
$$

which shows indeed that $\tau_{1}$ is the unique (upto a scalar factor) tau-function corresponding to $\psi_{1}$.

We will now introduce like in [19] and [20] elementary Bäcklund-Darboux transformations. They will act on wavefunctions of type $z^{\ell}$ and produce new wavefunctions of type $z^{\ell \pm 1}$. For $q, r \in R$, one considers the first order differential operators $q \partial q^{-1}$ and $r^{-1} \partial r$. An obvious question now is: When are $q \partial q^{-1} \psi$ and $r^{-1} \partial^{-1} r \psi$ again wavefunctions? The answer is given by the following proposition. But first notice that $r^{-1} \partial^{-1} r \psi$ is well and uniquely defined. If $\psi=P(z) e^{\sum t_{i} z^{i}}=P(\partial) e^{\sum t_{i} z^{i}}$, then

$$
\begin{aligned}
r^{-1} \partial^{-1} r \psi & =r^{-1} \partial^{-1} r P(\partial) e^{\sum t_{i} z^{i}} \\
& =r^{-1} \sum_{k=0}^{\infty}(-)^{k} \frac{\partial^{k}(r P(\partial))}{\partial x^{k}} \partial^{-k-1} e^{\sum t_{i} z^{i}} \\
& =r^{-1} \sum_{k=0}^{\infty}(-)^{k} \frac{\partial^{k}(r P(z))}{\partial x^{k}} z^{-k-1} e^{\sum t_{i} z^{i}} .
\end{aligned}
$$

Proposition 6.1. Let $\psi$ be a wavefunction of type $z^{\ell}$ and let $\psi^{*}$ be its adjoint wave function. Then the following holds:

(a) Let $q$ and $r \in R$ satisfy

$$
\begin{aligned}
& \partial_{n}(q)=\left(L^{n}\right)_{+}(q), \\
& \partial_{n}(r)=-\left(L^{n}\right)_{+}^{*}(r) \quad \text { for all } \quad n=1,2, \ldots
\end{aligned}
$$

Then $q \partial q^{-1} \psi\left(\right.$ resp. $\left.r^{-1} \partial^{-1} r \psi\right)$ is a wavefunction of type $z^{\ell+1}\left(\right.$ resp. $\left.z^{\ell-1}\right)$ and $-q^{-1} \partial^{-1} q \psi^{*}$ (resp. $\left.-r \partial r^{-1} \psi^{*}\right)$ is its adjoint wavefunction.

(b) If $q \partial q^{-1} \psi$ (resp. $\left.r^{-1} \partial^{-1} r \psi\right)$ is a wavefunction and hence $-q^{-1} \partial^{-1} q \psi^{*}$ (resp. $\left.-r \partial r^{-1} \psi^{*}\right)$ is its adjoint wavefunction, then one can find an up to a scalar multiple unique $\hat{q}$ (resp. $\hat{r}) \in R$ such that

$$
q \partial q^{-1}=\hat{q} \partial \hat{q}^{-1} \quad\left(\text { resp. } r^{-1} \partial r=\hat{r}^{-1} \partial \hat{r}\right),
$$


which satisfies the equations (6.3) (resp. (6.4)).

We say that $q$ (resp. $r$ ) is an eigenfunction (resp. adjoint eigenfunction) of the Lax operator $L$ if it satisfies (6.3) (resp. (6.4)).

Introduce for convenience of notation the operators

$$
P_{q}:=q \partial q^{-1} P \quad \text { and } \quad P_{r}:=r^{-1} \partial^{-1} r P .
$$

To prove part (a) of Proposition 6.1, one has to show that the $P_{q}$ and the $P_{r}$ satisfy the Sato-Wilson equations. To do so, we need some properties of the ring $R\left[\partial, \partial^{-1}\right.$ ) of pseudodifferential operators with coefficients from $R$. We resume them in a lemma.

Lemma 6.1. If $f$ belongs to $R$ and $Q$ to $R\left[\partial, \partial^{-1}\right)$, then the following identities hold

(a) $(Q f)_{-}=Q_{-} f$

(b) $(f Q)_{-}=f Q_{-}$,

(c) $\operatorname{Res}_{\partial}(Q f)=\operatorname{Res}_{\partial}(f Q)=f \operatorname{Res}_{\partial}(Q)$,

(d) $(\partial Q)_{-}=\partial Q_{-}-\operatorname{Res}_{\partial}(Q)$,

(e) $(Q \partial)_{-}=Q_{-} \partial-\operatorname{Res}_{\partial}(Q)$,

(f) $\left(Q \partial^{-1}\right)_{-}=Q_{-} \partial^{-1}+\operatorname{Res}_{\partial}\left(Q \partial^{-1}\right) \partial^{-1}$,

(g) $\left(\partial^{-1} Q\right)_{-}=\partial^{-1} Q_{-}+\partial^{-1} \operatorname{Res}_{\partial}\left(Q^{*} \partial^{-1}\right)$,

(h) $\operatorname{Res}_{\partial}\left(Q f \partial^{-1}\right)=Q_{+}(f)$,

(i) $\operatorname{Res}_{\partial}\left(\partial^{-1} f Q\right)=Q_{+}^{*}(f)$.

Since the proof of this lemma consists of straight forward calculations, we leave this to the reader.

If we denote $\partial /\left(\partial t_{n}\right)$ by $\partial_{n}$, then we get for $P_{q}=q \partial q^{-1} P$ that

$$
\begin{aligned}
\partial_{n}\left(P_{q}\right) P_{q}^{-1} & =\partial_{n}\left(q \partial q^{-1}\right) q \partial^{-1} q^{-1}+q \partial q^{-1} \partial_{n}(P) P^{-1} q \partial^{-1} q^{-1} \\
& =-q \partial q^{-1}\left(L^{n}\right)_{-} q \partial^{-1} q^{-1}+\partial_{n}\left(q \partial q^{-1}\right) q \partial^{-1} q^{-1} .
\end{aligned}
$$

Now we apply successively the identities from Lemma 6.1 to the first operator 
of the right-hand side

$$
\begin{aligned}
q \partial q^{-1}\left(L^{n}\right)_{-} q \partial^{-1} q^{-1}= & q \partial\left(q^{-1} L^{n} q\right)_{-} \partial^{-1} q^{-1} \\
= & q \partial\left(q^{-1} L^{n} q \partial^{-1}\right)_{-} q^{-1}-q \partial \operatorname{Res}_{\partial}\left(q^{-1} L^{n} q \partial^{-1}\right) \partial^{-1} q^{-1} \\
= & q\left(\partial q^{-1} L^{n} q \partial^{-1}\right)_{-} q^{-1}+q \operatorname{Res}_{\partial}\left(q^{-1} L^{n} q \partial^{-1}\right) q^{-1} \\
& -q \partial \operatorname{Res}_{\partial}\left(q^{-1} L^{n} q \partial^{-1}\right) \partial^{-1} q^{-1} \\
= & \left(q \partial q^{-1} L^{n} q \partial^{-1} q^{-1}\right)_{-}+q^{-1} \operatorname{Res}_{\partial}\left(L^{n} q \partial^{-1}\right) \\
& -q \partial q^{-1} \operatorname{Res}_{\partial}\left(L^{n} q \partial^{-1}\right) \partial^{-1} q^{-1}\left(q \partial q^{-1} L^{n} q \partial^{-1} q^{-1}\right)_{-} \\
& +\left(L^{n}\right)_{+}(q) q^{-1}-q \partial q^{-1}\left(L^{n}\right)_{+}(q) \partial^{-1} q^{-1} .
\end{aligned}
$$

On the other hand

$$
\partial_{n}\left(q \partial q^{-1}\right) q \partial^{-1} q^{-1}=\partial_{n}(q) q^{-1}-q \partial q^{-2} \partial_{n}(q) q \partial^{-1} q^{-1} .
$$

Thus we see that, if $\partial_{n}(q)=\left(L^{n}\right)_{+}(q)$, the operator $P_{q}$ satisfies the Sato-Wilson equation (2.2).

The proof that $P_{r}$ also satisfies the Sato-Wilson equation can be shown in a similar fashion.

To prove part (b) of Proposition 6.1, we first show uniqueness of the eigenfunction. Let $q_{1}$ and $q_{2}$ be two eigenfunctions that give the same BäcklundDarboux transformation, then

$$
\partial\left(\frac{q_{1}}{q_{2}}\right)=\frac{q_{1}}{q_{2}} \frac{\partial\left(q_{1}\right)}{q_{1}}-\frac{q_{1}}{q_{2}} \frac{\partial\left(q_{2}\right)}{q_{2}}=0,
$$

So $\partial^{k}\left(q_{1} / q_{2}\right)=0$ and $\partial^{k}\left(q_{1}\right)=\partial^{k}\left(\left(q_{1} / q_{2}\right) q_{2}\right)=\left(q_{1} / q_{2}\right) \partial^{k}\left(q_{2}\right)$. Now let $B_{\ell}=$ $\left(L^{\ell}\right)_{+}$, then

$$
\begin{aligned}
\partial_{n}\left(\frac{q_{1}}{q_{2}}\right) & =\frac{B_{n}\left(q_{1}\right)}{q_{2}}-\frac{q_{1}}{q_{2}^{2}} B_{n}\left(q_{2}\right) \\
& =\frac{1}{q_{2}} B_{n}\left(\frac{q_{1}}{q_{2}} q_{2}\right)-\frac{q_{1}}{q_{2}^{2}} B_{n}\left(q_{2}\right) \\
& =\frac{q_{1}}{q_{2}^{2}} B_{n}\left(q_{2}\right)-\frac{q_{1}}{q_{2}^{2}} B_{n}\left(q_{2}\right) \\
& =0
\end{aligned}
$$

for all $n=1,2, \ldots$ So we conclude that $q_{1}=\lambda q_{2}$ for a certain $\lambda \in \mathbb{C}$. Uniqueness for $r$ follows analogously.

Suppose now $q \partial q^{-1} \psi$ and $r^{-1} \partial^{-1} r \psi$ are again wavefunctions, but $q$ is not an eigenfunction and $r$ is not an adjoint eigenfunction. Since $P_{q}$ and $P_{r}$ satisfy 
the Sato-Wilson equation,

$$
\begin{gathered}
\partial_{n}(q)-\left(L^{n}\right)_{+}(q)-q \partial q^{-1}\left\{\partial_{n}(q)-\left(L^{n}\right)_{+}(q)\right\} \partial^{-1}=0, \\
\partial_{n}(r)+\left(L^{n}\right)_{+}^{*}(r)-\partial\left\{\partial_{n}(r)+\left(L^{n}\right)_{+}^{*}(r)\right\} r^{-1} \partial r=0
\end{gathered}
$$

and thus

$$
\begin{aligned}
& \partial\left(q^{-1}\left\{\partial_{n}(q)-\left(L^{n}\right)_{+}(q)\right\}\right)=0 \\
& \partial\left(r^{-1}\left\{\partial_{n}(r)+\left(L^{n}\right)_{+}^{*}(r)\right\}\right)=0 .
\end{aligned}
$$

So

$$
\begin{aligned}
& \partial_{n}(q)-\left(L^{n}\right)_{+}(q)=f_{n}\left(t_{2}, t_{3}, \ldots\right) q, \\
& \partial_{n}(r)+\left(L^{n}\right)_{+}^{*}(r)=g_{n}\left(t_{2}, t_{3}, \ldots\right) r .
\end{aligned}
$$

Let again $B_{\ell}=\left(L^{\ell}\right)_{+}$, and write down the compatibility conditions for $q$, i.e., $\partial_{n}\left(\partial_{\ell}(q)=\partial_{\ell}\left(\partial_{n}(q)\right)\right.$. One has

$$
\begin{aligned}
\partial_{n}\left(\partial_{\ell}(q)\right) & =\partial_{n}\left(B_{\ell}(q)+f_{\ell} q\right) \\
& =\partial_{n}\left(B_{\ell}\right)(q)+B_{\ell}\left(\partial_{n}(q)\right)+\partial_{n}\left(f_{\ell}\right) q+f_{\ell} \partial_{n}(q) \\
& =\partial_{n}\left(B_{\ell}\right)(q)+B_{\ell} B_{n}(q)+B_{\ell}\left(f_{n} q\right)+\partial_{n}\left(f_{\ell}\right) q+f_{\ell} B_{n}(q)+f_{\ell} f_{n} q
\end{aligned}
$$

and a similar relation with $n$ and $\ell$ interchanged, so we find

$$
\begin{gathered}
\left(\partial_{n}\left(B_{\ell}\right)-\partial_{\ell}\left(B_{n}\right)+B_{\ell} B_{n}-B_{n} B_{\ell}\right)(q)+B_{\ell}\left(f_{n} q\right)-B_{n}\left(f_{\ell} q\right) \\
+f_{\ell} B_{n}(q)-f_{n} B_{\ell}(q)+\partial_{n}\left(f_{\ell}\right) q-\partial_{\ell}\left(f_{n}\right) q=0 .
\end{gathered}
$$

Because the Zakharov-Shabat equations (1.5) hold for the $B_{\ell}$ 's and $f_{m}$ is independent of $x=t_{1}$ one finds that

$$
\partial_{n}\left(f_{\ell}\right)=\partial_{\ell}\left(f_{n}\right)
$$

and hence it is formally possible to integrate to all times $t_{j}$. So one can find a function $F(t)$ such that

$$
f_{n}(t)=F(t)^{-1} \partial_{n} F(t)
$$

Now choose

$$
\hat{q}=F(t)^{-1} q,
$$

then $\partial_{n}(\hat{q})=B_{n}(\hat{q})$. A similar argument holds for $r$. This concludes the proof of Proposition 6.1. 
In our setting it is easy to find (adjoint) eigenfunctions and hence elementary Bäcklund-Darboux transformations. In fact one can construct for each point of the Grassmannian all elementary Bäcklund-Darboux transformations. Let $\psi_{W}$ be a wavefunction of type $z^{\ell}$, corresponding to $W \in G r^{(\ell)}(H)$ and let $\psi_{W}^{*}$ be its adjoint wavefunction. Recall from Section 3 that its adjoint wavefunction spans $W^{\perp}$ with respect to the bilinear form $B(\cdot, \cdot)$ of $(3.1)$. For convenience of notation we replace this bilinear form by $\langle\cdot \mid \cdot\rangle$ defined in (5.1). Choose $s(z) \in H$, respectively, then clearly

$$
q(t):=\left\langle\psi_{W}(t, z) \mid s(z)\right\rangle, \quad \text { respectively } r(t):=\left\langle\psi_{W}^{*}(t, z) \mid s(z)\right\rangle
$$

is an eigenfunction, respectively adjoint eigenfunction. Assume now that $q(t) \neq$ 0 and $r(t) \neq 0$, then by Proposition 6.1

$$
\begin{array}{ll}
\psi_{q}=q \partial q^{-1} \psi_{W}, & \psi_{r}=r^{-1} \partial^{-1} r \psi_{W}, \\
\psi_{q}^{*}=-q^{-1} \partial^{-1} q \psi_{W}^{*}, & \psi_{r}^{*}=-r \partial r^{-1} \psi_{W}^{*}
\end{array}
$$

are wavefunctions, respectively adjoint wavefunction. Now assume that $\psi_{q}$, $\psi_{r}, \psi_{q}^{*}, \psi_{r}^{*}$, respectively corresponding to the new point $W_{q}, W_{r}, W_{q}^{\perp}, W_{r}^{\perp}$ of $G r(H)$. Since $q \partial q^{-1}$ and $-r \partial r^{-1}$ are first order differential operators we find that $W_{q} \subset W$ and $W_{r}^{\perp} \subset W^{\perp}$. Moreover,

$$
\begin{aligned}
\left\langle\psi_{q}(t, z) \mid s(z)\right\rangle & =\left\langle\frac{\partial \psi_{W}(t, z)}{\partial x}-q(t)^{-1} \frac{\partial q(t)}{\partial x} \psi_{W}(t, z) \mid s(z)\right\rangle \\
& =\left\langle\frac{\partial \psi_{W}(t, z)}{\partial x} \mid s(z)\right\rangle-\left\langle\frac{\partial \psi_{W}(t, z)}{\partial x} \mid s(z)\right\rangle=0
\end{aligned}
$$

and

$$
\begin{aligned}
\left\langle\psi_{r}^{*}(t, z) \mid s(z)\right\rangle & =\left\langle\frac{\partial \psi_{W}^{*}(t, z)}{\partial x}+r(t)^{-1} \frac{\partial r(t)}{\partial x} \psi_{W}^{*}(t, z) \mid s(z)\right\rangle \\
& =\left\langle\frac{\partial \psi_{W}^{*}(t, z)}{\partial x} \mid s(z)\right\rangle-\left\langle\frac{\partial \psi_{W}^{*}(t, z)}{\partial x} \mid s(z)\right\rangle=0 .
\end{aligned}
$$

Hence $\left\langle W_{q} \mid s(z)\right\rangle=\left\langle W_{r}^{\perp} \mid s(z)\right\rangle=0$. Next assume that also $u(z) \in H$, be such that

$$
\left\langle\psi_{q}(t, z) \mid u(z)\right\rangle=0 \quad \text { but }\left\langle\psi_{W}(t, z) \mid u(z)\right\rangle \neq 0,
$$

then one gets that

$$
\frac{\left\langle\frac{\partial \psi_{W}(t, z)}{\partial x} \mid u(z)\right\rangle}{\left\langle\psi_{W}(t, z) \mid u(z)\right\rangle}=\frac{\frac{\partial q(t)}{\partial x}}{q(t)} .
$$


Since both $q(t)$ and $\left\langle\psi_{q}(t, z) \mid u(z)\right\rangle$ are eigenfunctions that by (6.9) give the same Bäcklund-Darboux transformation, one finds

$$
q(t)=\lambda\left\langle\psi_{q}(t, z) \mid u(z)\right\rangle
$$

and hence $\left\langle\psi_{W}(t, z) \mid s(z)-\lambda u(z)\right\rangle=0$. From this we conclude that $W_{q} \subset W$ is a codimension 1 inclusion. A similar argument gives that $W_{r}^{\perp} \subset W^{\perp}$ is also a codimension 1 inclusion. Notice that for two arbitrary points $W$ and $W_{q}$ of the Grassmannian such that $W_{q} \subset W$ is a codimension 1 , with wavefunctions $\psi_{W}(t, z)$ and $\psi_{q}(t, z)$ one can always find a function $u(z) \in H$ such that $W_{q}=$ $\{w \in W \mid\langle w(z) \mid u(z)\rangle=0\}$. Hence, our method produces all possible elementary Bäcklund-Darboux transformations.

Next we want to determine the tau-functions corresponding to an elementary Bäcklund-Darboux transformations constructed from (6.8).

Let $q(t) \partial q(t)^{-1}$ (resp. $-r(t) \partial r(t)^{-1}$ ) act on the wavefunction $\psi_{W}(t, z)$ (resp. adjoint wavefunction $\psi_{W}^{*}(t, z)$ and compare this with $\psi_{q}(t, z)$ (resp. $\left.\psi_{r}^{*}(t, z)\right)$. We thus obtain

$$
\begin{gathered}
z P_{W_{q}}(t, z)=\frac{\partial P_{W}(t, z)}{\partial x}+\left(z-q^{-1} \frac{\partial q}{\partial x}\right) P_{W}(t, z) \\
z P_{W_{r}}^{*-1}(t,-z)=-\frac{\partial P_{W}^{*-1}(t,-z)}{\partial x}+\left(z+r^{-1} \frac{\partial r}{\partial x}\right) P_{W}^{*-1}(t,-z)
\end{gathered}
$$

Comparing the coefficients of $z^{ \pm \ell}$ we find that

$$
\frac{\partial \log q}{\partial x}=\frac{\partial \log \tau_{W_{q}} / \tau_{W}}{\partial x} \text { and } \quad \frac{\partial \log r}{\partial x}=\frac{\partial \log \tau_{W_{r}} / \tau_{W}}{\partial x} .
$$

This suggests the following relations

$$
\tau_{W_{q}}(t)=\lambda q(t) \tau_{W}(t) \text { and } \quad \tau_{W_{r}}(t)=\mu r(t) \tau_{W}(t),
$$

with $\lambda, \mu \in \mathbb{C}^{\times}$. Since the tau-function of a plane $W$ in $\operatorname{Gr}(H)$ is only determined up to a constant, one may assume that the constants $\lambda$ and $\mu$ are equal to 1 . Since $\tau_{W}$ is a tau-function, it satisfies the bilinear identity (4.3).

Now let the following operator

$$
u^{\ell} e^{\sum_{i=1}^{\infty} t_{i} u^{i}+s_{i} w^{i}} e^{-\sum_{i=1}^{\infty}\left(\frac{\partial}{\partial t_{i}} \frac{w^{-i}}{i}+\frac{\partial}{\partial s_{i}}\right) \frac{w^{-i}}{i}}
$$

act on this bilinear identity. Then we obtain after some calculation that

$$
\begin{aligned}
& \operatorname{Res}_{z} u^{\ell} \tau_{W}\left(t-\left[z^{-1}\right]-\left[u^{-1}\right]\right) e^{\sum\left(t_{i}-\frac{z^{i}}{i}\right) u^{i}} \\
& e^{\sum\left(t_{i}-s_{i}\right) z^{i}} w^{\ell} \tau_{W}\left(s-\left[w^{-1}\right]+\left[z^{-1}\right]\right) e^{\sum\left(s_{i}+\frac{z^{i}}{i}\right) w^{i}}=0 .
\end{aligned}
$$


Multiply with $s(u) s(w)$ and take the residues at $u=0, w=0$, then

$$
\operatorname{Res}_{z} q\left(t-\left[z^{-1}\right]\right) \tau_{W}\left(t-\left[z^{-1}\right]\right) e^{\sum\left(t_{i}-s_{i}\right) z^{i}} q\left(t+\left[z^{-1}\right]\right) \tau_{W}\left(t+\left[z^{-1}\right]\right)=0
$$

Hence

$$
\tau_{q}:=q(t) \tau_{W}(t)
$$

is again a tau-function, its corresponding wave function of type $z^{\ell+1}$ is

$$
\begin{aligned}
\tilde{\psi}_{q}(t, z) & =z^{\ell+1} \frac{q\left(t-[z]^{-1}\right)}{q(t)} \frac{\tau_{W}\left(t-\left[z^{-1}\right]\right)}{\tau_{W}(t)} e^{\sum t_{i} z^{i}} \\
& =z \frac{q\left(t-[z]^{-1}\right)}{q(t)} \psi_{W}(t, z) .
\end{aligned}
$$

Since, $q(t)=\tau_{q}(t) /\left(\tau_{W}(t)\right)$ and $q$ is an eigenfunction, $\tilde{\psi}_{q}(t, z)$ must be equal to

$$
\begin{aligned}
\tilde{\psi}_{q}(t, z) & =\frac{\partial \psi_{W}(t, z)}{\partial x}-\frac{\partial \log \left(\frac{\tau_{q}(t)}{\tau_{W}(t)}\right)}{\partial x} \psi_{W}(t, z) \\
& =q(t) \partial q(t)^{-1} \psi_{W}(t, z)=\psi_{q}(t, z) .
\end{aligned}
$$

Now use formula (4.2) and one obtains that

$$
\partial_{\ell} \log \frac{\tau_{W_{q}}(t)}{q(t) \tau_{W}(t)}=0 \quad \text { for all } \quad \ell=1,2, \ldots
$$

Thus we conclude that upto multiplication by a constant

$$
\tau_{W_{q}}(t)=q(t) \tau_{W}(t)
$$

The formula $\tau_{W_{r}}(t)=r(t) \tau_{W}(t)$ can be obtained in a similar matter.

We now resume the results on elementary Bäcklund-Darboux transformations in the following

Theorem 6.1. Let $W \in G r(H)$ and $\psi_{W}(t, z), \psi_{W}^{*}(t, z), \tau_{W}(t)$ the to $W$ and $W^{\perp}$ corresponding wavefunction, adjoint wavefunction and taufunction. Let $s \in H$ be such that $q(t)=\left\langle\psi_{W}(t, z) \mid s(z)\right\rangle \neq 0$ (respectively $\left.r(t)=\left\langle\psi_{W}^{*}(t, z) \mid s(z)\right\rangle \neq 0\right)$. Then $q(t) \partial q(t)^{-1}$ (resp. $\left.r(t)^{-1} \partial r(t)\right)$ define ele- 
mentary Bäcklund-Darboux transformations such that

$$
\begin{aligned}
\psi_{q}(t, z) & =q(t) \partial q(t)^{-1} \psi_{W}(t, z)=z \frac{q\left(t-\left[z^{-1}\right]\right)}{q(t)} \psi_{W}(t, z), \\
\psi_{q}^{*}(t, z) & =-q(t)^{-1} \partial^{-1} q(t) \psi_{W}^{*}(t, z)=z^{-1} \frac{q\left(t+\left[z^{-1}\right]\right)}{q(t)} \psi_{W}^{*}(t, z), \\
\tau_{q}(t) & =q(t) \tau_{W}(t), \text { respectively } \\
\psi_{r}(t, z) & =r(t)^{-1} \partial^{-1} r(t) \psi_{W}(t, z)=z^{-1} \frac{r\left(t-\left[z^{-1}\right]\right)}{r(t)} \psi_{W}(t, z), \\
\psi_{r}(t, z)^{*} & =-r(t) \partial r(t)^{-1} \psi_{W}^{*}(t, z)=z \frac{r\left(t+\left[z^{-1}\right]\right)}{r(t)} \psi_{W}^{*}(t, z), \\
\tau_{r}(t) & =r(t) \tau_{W}(t)
\end{aligned}
$$

are the new wavefunction, adjoint wavefunction and tau-function corresponding to

$$
W_{q}=\{w \in W \mid\langle w(z) \mid s(z)\rangle=0\} \quad \text { and } \quad W_{q}^{\perp}=W^{\perp} \oplus \mathbb{C} s,
$$

respectively

$$
W_{r}=W \oplus \mathbb{C} s \quad \text { and } \quad W_{r}^{\perp}=\left\{w \in W^{\perp} \mid\langle w(z) \mid s(z)\rangle=0\right\} .
$$

\section{§7. Squared Eigenfunction Potentials}

Let $q$ be an eigenfunction and $r$ be an adjoint eigenfunction of the KP Lax operator $L$. For such a pair of (adjoint) eigenfunctions Oevel [19] showed that there exists a function $\Omega(r, q)$ called the squared eigenfunction potential, which satisfies

$$
\frac{\partial \Omega(r, q)}{\partial t_{k}}=\operatorname{Res}_{\partial}\left(\partial^{-1} r\left(L^{k}\right)_{+} q \partial^{-1}\right) .
$$

This potential was described in [17] and [7] (see also [19] and [20]). A special case of (7.1) is the case that $k=1$, i.e. $t_{1}=x$, then

$$
\frac{\partial \Omega(r, q)}{\partial x}=q r
$$

Equation (7.1) determines $\Omega$ uniquely upto a shift by a constant.

The expressions $\partial^{-1} r \psi$ and $\partial^{-1} \psi^{*} q$ are special squared eigenfunction potentials. Notice that they are uniquely defined. We now want to find an explicit expression for these specific potentials. Since

$$
\psi_{r}=r^{-1} \partial^{-1} r \psi_{W} \quad \text { and } \quad \psi_{q}^{*}=-q^{-1} \partial^{-1} q \psi_{W},
$$


one has

$$
\begin{aligned}
\partial^{-1} r(t) \psi_{W}(t, z) & =r(t) \psi_{r}(t, z) \\
& =r(t) z^{-1} \frac{r\left(t-\left[z^{-1}\right]\right)}{r(t)} \psi_{W}(t, z) \\
& =z^{-1} r\left(t-\left[z^{-1}\right]\right) \psi_{W}(t, z)
\end{aligned}
$$

and similarly $\partial^{-1} q(t) \psi_{W}^{*}(t, z)=-z^{-1} q\left(t+\left[z^{-1}\right]\right) \psi_{W}^{*}(t, z)$. We can also express them in $s(z)$. Let as before,

$$
q(t)=\left\langle\psi_{W}(t, z) \mid s(z)\right\rangle, \quad \text { and } \quad r(t)=\left\langle\psi_{W}^{*}(t, z) \mid s(z)\right\rangle,
$$

then $\partial^{-1} r \psi$ and $\partial^{-1} \psi^{*} q$ are equal to

$$
\begin{aligned}
& S\left(\psi_{W}(t, z), s(z)\right):=z^{-1}\left\langle\psi_{W}^{*}\left(t-\left[z^{-1}\right], y\right) \mid s(y)\right\rangle \psi_{W}(t, z) \\
& S\left(\psi_{W}^{*}(t, z), s(z)\right):=-z^{-1} \psi_{W}^{*}(t, z)\left\langle\psi_{W}\left(t+\left[z^{-1}\right], y\right) \mid s(y)\right\rangle
\end{aligned}
$$

respectively.

Next let $W \in G r^{(\ell)}(H)$, then, since $\psi_{W}(t, z)$ and $\psi_{W}^{*}(t, z)$ satisfy the formula of Proposition 4.1 we find the following expressions for these squared eigenfunction potentials (see also [4]).

Proposition 7.1. Let $|z|>|y|$, then for $W \in G r^{(\ell)}(H)$ on has

$$
\begin{aligned}
& S\left(\psi_{W}(t, z), s(z)\right)=\frac{\left\langle(z / y)^{\ell} X(t, z, y) \tau_{W}(t) \mid s(y)\right\rangle}{\tau_{W}(t)}, \\
& S\left(\psi_{W}^{*}(t, z), s(z)\right)=\frac{\left\langle(y / z)^{\ell} X(t, y, z) \tau_{W}(t) \mid s(y)\right\rangle}{\tau_{W}(t)},
\end{aligned}
$$

where

$$
X(t, \lambda, \mu)=\frac{1}{\lambda-\mu} \exp \left(\sum t_{i}\left(\lambda^{i}-\mu^{i}\right)\right) \exp \left(\sum \frac{\mu^{-i}-\lambda^{-i}}{i} \frac{\partial}{\partial t_{i}}\right)
$$

is the vertex operator for the KP-hierarchy.

This vertex operator expresses the action of the Lie algebra $g l_{\infty}$, see [14] for more details, it also is a generating series of the $W_{1+\infty}$ algebra.

\section{$\S 8$. Proof of the Main Theorem}

The following Lemma from [21] will be usefull in the proof of the Main Theorem. 
Lemma 8.1. Let $Q=\partial^{n}+a_{n-1} \partial^{n-1}+\cdots+a_{1} \partial+a_{0}$ be an $n$-th order monic differential operator with coefficients in $R$ and let $f_{1}, \ldots, f_{n} \in \operatorname{Ker} Q$, linearly independent functions in $R$. Let $\left(b_{1}, b_{2}, \ldots, b_{n}\right)$ be the solution of the linear system

$$
\left(\begin{array}{cccc}
f_{1} & f_{2} & \cdots & f_{n} \\
\frac{\partial f_{1}}{\partial x} & \frac{\partial f_{2}}{\partial x} & \cdots & \frac{\partial f_{n}}{\partial x} \\
\vdots & \vdots & \ddots & \vdots \\
\frac{\partial^{n-2} f_{1}}{\partial x^{n-2}} & \frac{\partial^{n-2} f_{2}}{\partial x^{n-2}} & \cdots & \frac{\partial^{n-2} f_{n}}{\partial x^{n-2}} \\
\frac{\partial^{n-1} f_{1}}{\partial x^{n-1}} & \frac{\partial^{n-1} f_{2}}{\partial x^{n-1}} & \cdots & \frac{\partial^{n-1} f_{n}}{\partial x^{n-1}}
\end{array}\right)\left(\begin{array}{c}
b_{1} \\
b_{2} \\
\vdots \\
b_{n-1} \\
b_{n}
\end{array}\right)=\left(\begin{array}{c}
0 \\
0 \\
\vdots \\
0 \\
1
\end{array}\right) .
$$

Then

$$
Q^{-1}=\sum_{k=1}^{n} f_{k} \partial^{-1} b_{k}
$$

Proof (cf. [21]). We use the fact that for $f \in R$

$$
f \partial^{-1}=-\left(\partial^{-1} f\right)^{*}=\sum_{\ell=0}^{\infty}\left((-)^{\ell+1} \frac{\partial^{\ell} f}{\partial x^{\ell}} \partial^{-\ell-1}\right)^{*}=\sum_{\ell=0}^{\infty} \partial^{-\ell-1} \frac{\partial^{\ell} f}{\partial x^{\ell}}
$$

and calculate

$$
\begin{aligned}
Q \sum_{k=1}^{n} f_{k} \partial^{-1} b_{k} & =\left(Q \sum_{k=1}^{n} f_{k} \partial^{-1} b_{k}\right)_{+}+\left(Q \sum_{k=1}^{n} f_{k} \partial^{-1} b_{k}\right)_{-} \\
& =\left(Q \sum_{\ell=0}^{\infty} \partial^{-\ell-1} \sum_{k=1}^{n} \frac{\partial^{\ell} f_{k}}{\partial x^{\ell}} b_{k}\right)_{+}+\sum_{k=1}^{n} Q\left(f_{k}\right) \partial^{-1} b_{k} \\
& =1 .
\end{aligned}
$$

This finishes the proof of the lemma.

We now want to generalize the situation of Theorem 6.1 and proof the Main Theorem of this paper, Theorem 5.1. Assume that is given the plane $W \in G r^{(\ell)}(H)$ with wavefunction $\psi_{W}(t, z)$ adjoint wavefunction $\psi_{W}^{*}(t, z)$ and tau-function $\tau_{W}$. We want to determine the Bäcklund-Darboux transformation $B D(V, W)$ for $W \in G r(H)$ that maps $\psi_{W}(t, z)$ to $\psi_{V}(t, z)$ (cf. [15]). We restrict ourselves to the case that $B D(V, W)$ is a finite product of elementary BäcklundDarboux transformation, i.e., to the case that $V \cap W$ has finite codimension inside both $V$ and $W$. Of course one has that if $\psi_{W}(t, z)=P_{W}(\partial) e^{\sum t_{i} z^{i}}$ and 
$\psi_{V}(t, z)=P_{V}(\partial) e^{\sum t_{i} z^{i}}$, that $B D(V, W)=P_{V}(\partial) P_{W}(\partial)^{-1}$. But we assume that the wavefunction and its adjoint are only given for $W$ and not yet known for $V$.

Proof of Theorem 5.1. Recall that $V \cap W$ has codimension $m$ in $V$ and codimension $n$ in $W$ and that we take elements $w_{1}, w_{2}, \ldots, w_{n}$ in $V^{\perp}$ such that

$$
V=\left\{x \mid x \in V+W,\left\langle x \mid w_{i}\right\rangle=0 \text { for all } i\right\}
$$

and elements $v_{1}, v_{2}, \ldots, v_{m}$ in $V+W$ such that

$$
(V+W)^{\perp}=\left\{x \mid x \in W^{\perp},\left\langle x \mid v_{j}\right\rangle=0 \text { for all } j\right\} .
$$

Starting with $W$ we first apply $m$ elementary adjoint Bäcklund-Darboux transformations to obtain $W+V$ and then apply $n$ elementary Bäcklund-Darboux transformations to obtain $V$. In other words

$$
B D(V, W)=B D(V, V+W) B D(V+W, W) .
$$

Notice first that $B D(V, V+W)$ the product is of $n$ operators of the form $q_{i} \partial q_{i}^{-1}$, hence it is a differential operator of order $n$ for which the leading coefficient is equal to 1 .

Since $B D(V+W, W)$ is the product of $m$ elementary adjoint BäcklundDarboux transformations $r_{i}^{-1} \partial^{-1} r_{i}$, one finds that $(-1)^{m} B D(V+W, W)^{*-1}$ is a differential operator of order $m$, say $\sum_{k=0}^{m} a_{k} \partial^{k}$, with $a_{m}=1$. Then

$$
\psi_{V+W}^{*}=\sum_{k=0}^{m} a_{k} \frac{\partial^{k} \psi_{W}^{*}}{\partial x^{k}}
$$

and $\left\langle\psi_{V+W}^{*} \mid v_{i}\right\rangle=0$ for all $1 \leq i \leq m$. Thus for all $1 \leq i \leq m$ the $\left\langle\psi_{W}^{*} \mid v_{i}\right\rangle \in$ Ker $(-1)^{m} B D(V+W, W)^{*-1}$ and they are linearly independent. So we can apply Lemma 8.1, this gives that

$$
(-1)^{m} B D(V+W, W)^{*}=\sum_{k}(-1)^{m}\left\langle\psi_{W}^{*}(t, z) \mid v_{k}(z)\right\rangle \partial^{-1} d_{k},
$$

for certain $d_{k}$. Hence,

$$
B D(V+W, W)=\sum_{k=1}^{m} b_{k} \partial^{-1}\left\langle\psi_{W}^{*} \mid v_{k}\right\rangle
$$

is in fact a pseudodifferential operator of order $-m$, again with leading coefficient equal to 1 . Now multiplying the two Bäcklund-Darboux transformations, 
we obtain that

$$
\begin{aligned}
B D(V, W) & =\left(\sum_{\ell=0}^{n} c_{\ell} \partial^{\ell}\right)\left(\sum_{k=1}^{m} b_{k} \partial^{-1}\left\langle\psi_{W}^{*} \mid v_{k}\right\rangle\right) \\
& =\sum_{k=1}^{m} a_{-k} \partial^{-1}\left\langle\psi_{W}^{*} \mid v_{k}\right\rangle+\sum_{\ell=0}^{n-m} a_{\ell} \partial^{\ell}
\end{aligned}
$$

is a pseudodifferential operator of order $n-m$ with leading coefficient equal to 1 (notice that this is $a_{n-m}$ if $n \geq m$ ). Since $B D(V, W) \psi_{W}$ belongs to $V$ it must be perpendicular to $w_{1}, w_{2}, \ldots, w_{n}$, which gives the restriction

$$
\sum_{k=1}^{m} a_{-k} S\left(\psi_{W}, v_{k}, w_{j}\right)+\sum_{\ell=0}^{n-m} a_{\ell}\left\langle\frac{\partial^{\ell} \psi_{W}}{\partial x^{\ell}} \mid w_{j}\right\rangle=0,
$$

Where (cf. (7.2))

$$
S(A, s, t):=\langle S(A, s) \mid t\rangle .
$$

Now if $n \geq m$, the equations (8.2) determine $B D(V, W)$, since $a_{n-m}=1$. However, if $n<m$ then (8.2) is equal to

$$
\sum_{k=1}^{m} a_{-k} S\left(\psi_{W}, v_{k}, w_{j}\right)=0
$$

and we need some more information. We also know that $B D(V, W)$ is a pseudodifferential operator of order $n-m$ with leading coefficient equal to 1 . This leads to the extra restrictions.

$$
\sum_{k=1}^{m} a_{-k}\left\langle\frac{\partial^{j} \psi_{W}^{*}}{\partial x^{j}} \mid v_{k}\right\rangle=(-)^{m-n-1} \delta_{j, m-n-1} \quad \text { for } j=0,1, \ldots, m-n-1 .
$$

Then (8.4) and (8.5) determine $B D(V, W)$ uniquely. Using Cramer's rule we can determine in the case that $n \geq m$ from (8.2) and in the case that $m>n$ from (8.4) and (8.5) the coefficients $a_{i}$ of $B D(V, W)$. We thus obtain (cf. (5.2), $(5.3))$

$$
\begin{aligned}
B D(V, W)= & \mathcal{W}\left(\psi_{W} ; w_{1}, \ldots, w_{n} ; \psi_{W}^{*} ; v_{1}, \ldots, v_{m}\right)^{-1} \\
& \times \mathcal{W}\left(\psi_{W} ; w_{1}, \ldots, w_{n} ; \psi_{W}^{*} ; v_{1}, \ldots, v_{m} ; \partial\right) .
\end{aligned}
$$

Next, we want to calculate the inverse of $B D(V, W)$ or rather the adjoint of the inverse. First notice that

$$
\begin{aligned}
& B D(V, W)^{*-1}=B D\left(V^{\perp}, W^{\perp}\right) \text { and } \\
& B D(V, W)^{*-1}=B D(V, V \cap W)^{*-1} B D(V \cap W, W)^{*-1} .
\end{aligned}
$$


From the first line of (8.7) we deduce that

$$
\left\langle B D(V, W)^{*-1} \psi_{W}^{*} \mid v_{j}\right\rangle=0 \quad \text { for } \quad j=1,2, \ldots, m .
$$

Since $(-)^{m} B D(V, V \cap W)^{*-1}(B D(V \cap W, W))$ is an $m$-th, (resp. $n$-th) order monic differential operator and $\left\langle\psi_{W} \mid w_{\ell}\right\rangle \in \operatorname{Ker} B D(V \cap W, W)$ for all $1 \leq \ell \leq n$, we deduce from the second line that of (8.7)

$$
B D(V, W)^{*-1}=\sum_{\ell=1}^{n} a_{-\ell} \partial^{-1}\left\langle\psi_{W} \mid w_{\ell}\right\rangle+\sum_{k=0}^{m-n} a_{k} \partial^{k}
$$

Using the fact that $B D(V, W)^{*-1}$ is an $(m-n)$-th order pseudodifferential operator with leading coefficient $(-1)^{m-n}$ we obtain that for $1 \leq j \leq m$

$$
\sum_{\ell=1}^{n} a_{-\ell} S\left(\psi_{W}^{*}, w_{\ell}, v_{j}\right)+\sum_{k=0}^{m-n-1} a_{k}\left\langle\frac{\partial^{k} \psi_{W}^{*}}{\partial x^{k}} \mid v_{j}\right\rangle=(-1)^{n-m-1},
$$

if $m \geq n$ and that if $m<n$

$$
\begin{aligned}
& \sum_{\ell=1}^{n} a_{-\ell} S\left(\psi_{W}^{*}, w_{\ell}, v_{j}\right)=0, \\
& \sum_{\ell=1}^{n} a_{-\ell}\left\langle\frac{\partial^{k} \psi_{W}}{\partial x^{k}} \mid w_{\ell}\right\rangle=-\delta_{k, n-m-1},
\end{aligned}
$$

for $1 \leq j \leq m$ and $0 \leq k \leq n-m-1$. This determines $B D(V, W)^{*-1}$ uniquely. One has

$$
\begin{aligned}
B D(V, W)^{*-1}= & (-)^{n+m} \mathcal{W}\left(\psi_{W}^{*} ; v_{1}, \ldots, v_{m} ; \psi_{W} ; w_{1}, \ldots, w_{n}\right)^{-1} \\
& \times \mathcal{W}\left(\psi_{W}^{*} ; v_{1}, \ldots, v_{m} ; \psi_{W} ; w_{1}, \ldots, w_{n} ; \partial\right)
\end{aligned}
$$

We now want to calculate $\tau_{V}$. In order to do that we write $B D(V, W)$ as product of $n+m$ elementary Bäcklund-Darboux transformations

$$
B D(V, W)=B D\left(V, U_{n+m-1}\right) B D\left(U_{n+m-1}, U_{n+m-2}\right) \cdots B D\left(U_{1}, W\right),
$$

where

$$
U_{k}= \begin{cases}W+\sum_{j=1}^{k} \mathbb{C} v_{j} & \text { for } 1 \leq k \leq m, \\ \left\{u \in W+V \mid\left\langle u \mid w_{j}\right\rangle=0 \text { for } 1 \leq j \leq k-n\right\} & \text { for } m \leq k \leq m+n .\end{cases}
$$

Then from Theorem 6.1 we deduce that for $k \leq m$

$$
\begin{aligned}
\tau_{U_{k}} & =\left\langle\psi_{U_{k-1}}^{*} \mid v_{k}\right\rangle \tau_{U_{k-1}} \\
& =\left\langle B D\left(U_{k-1}, W\right)^{*-1} \psi_{W}^{*} \mid v_{k}\right\rangle \tau_{U_{k-1}} \\
& =(-)^{k-1} \frac{\mathcal{W}\left(\psi_{W}^{*} ; v_{1}, \ldots, v_{k}\right)}{\mathcal{W}\left(\psi_{W}^{*} ; v_{1}, \ldots, v_{k-1}\right)} \tau_{U_{k-1}}
\end{aligned}
$$


and that for $k>m$

$$
\begin{aligned}
\tau_{U_{k}} & =\left\langle\psi_{U_{k-1}} \mid w_{k-m}\right\rangle \tau_{U_{k-1}} \\
& =\left\langle B D\left(U_{k-1}, W\right) \psi_{W} \mid w_{k-m}\right\rangle \tau_{U_{k-1}} \\
& =\frac{\mathcal{W}\left(\psi_{W} ; w_{1}, \ldots, w_{k-m} ; \psi_{W}^{*} ; v_{1}, \ldots, v_{m}\right)}{\mathcal{W}\left(\psi_{W} ; w_{1}, \ldots, w_{k-m-1} ; \psi_{W}^{*} ; v_{1}, \ldots, v_{m}\right)} \tau_{U_{k-1}} .
\end{aligned}
$$

From which we deduce that (upto a scalar multiple)

$$
\tau_{V}=\mathcal{W}\left(\psi_{W} ; w_{1}, \ldots, w_{n} ; \psi_{W}^{*} ; v_{1}, \ldots, v_{m}\right) \tau_{W} .
$$

Thus we have proven the Theorem 5.1 of this paper.

\section{§9. Bäcklund-Darboux Transformations for the Constrained KP Hierarchies}

In this section we want to consider the elementary Bäcklund-Darboux transformations for the $m$-vector $n$-constrained KP hierarchy. These hierarchies are generalizations of the well-known Gelfand-Dickey- or $n$-th reduced $\mathrm{KP}$ hierarchy. Let $L$ be the KP Lax operator and let $q_{j}, r_{j}, 1 \leq j \leq m$ be eigenfunctions, respectiveley adjoint eigenfunctions, then $L$ belongs to the $m$ vector $n$-constrained KP hierarchy if $\left(L^{n}\right)_{-}=\sum_{j=1}^{m} q_{j} \partial^{-1} r_{j}$. Another form of these hierarchies were introduced by Krichever [16], see also Dickey [10], as the rational reductions of the KP hierarchy. Then $L^{n}=L_{2}^{-1} L_{1}$, where $L_{1}$ and $L_{2}$ are differential operators of order $m+n$, respectively $m$, satisfying:

$$
\begin{aligned}
& \partial_{i} L_{1}=\left(\left(L_{1} L_{2}^{-1}\right)^{i / n}\right)_{+} L_{1}-L_{1}\left(\left(L_{2}^{-1} L_{1}\right)^{i / n}\right)_{+}, \\
& \partial_{i} L_{2}=\left(\left(L_{1} L_{2}^{-1}\right)^{i / n}\right)_{+} L_{2}-L_{2}\left(\left(L_{2}^{-1} L_{1}\right)^{i / n}\right)_{+} .
\end{aligned}
$$

In [12] (see also [13]) the authors show that both descriptions define the same hierarchy. They use the Segal-Wilson Grassmannian to show that. Both hierarchies are characterized by points $W$ in the Grassmannian that have a subspace $W^{\prime}$ of codimension $m$ such that $z^{n} W^{\prime} \subset W$. This geometric description also holds for the Sato Grassmannian. We can construct this subspace $W^{\prime}$ as follows. Assume, without loss of generalization, that $m$ is minimal, then $z^{n} W \subset W+\sum_{j=1}^{m} \mathbb{C} s_{j}$, then one can find $m$ functions $u_{j}$ such that $\left\langle W \mid u_{j}\right\rangle=0$ and $\left\langle s_{i} \mid u_{j}\right\rangle=\delta_{i j}$. Then

$$
W^{\prime}=\left\{w \in W \mid\left\langle w \mid z^{n} u_{j}\right\rangle=0 \text { for all } 1 \leq j \leq m\right\} .
$$

The construction of elementary Bäcklund-Darboux transformations that preserve the $m$-vector $n$-constrained hierarchy can be deduced from the following Lemma. 
Lemma 9.1. Let $W^{\prime} \subset W$ such that $z^{n} W^{\prime} \subset W$ and let

$$
\begin{gathered}
W_{s}=\{w \in W \mid\langle w \mid s\rangle=0\}, \quad W_{s}^{\prime}=\left\{w \in W^{\prime} \mid\langle w \mid s\rangle=0\right\}, \\
W_{u}=W+\mathbb{C} u \quad \text { and } \quad W_{u}^{\prime}=W^{\prime}+\mathbb{C} u
\end{gathered}
$$

Then $z^{n} W_{s}^{\prime} \subset W_{s}\left(z^{n} W_{u}^{\prime} \subset W_{u}\right)$ if and only if there exists a $\lambda \in \mathbb{C}$ such that

$$
\left(z^{n}-\lambda\right) s \in W^{\prime \perp} \quad\left(\text { resp. }\left(z^{n}-\lambda\right) u \in W\right) .
$$

Proof. Let $v \in W_{s}^{\prime}$ and $z^{n} v \in W_{s}$, then

$$
\left\langle z^{n} v \mid s(z)\right\rangle=\left\langle v \mid z^{n} s(z)\right\rangle=0,
$$

in other words $z^{n} s(z) \in W_{s}^{\prime \perp}$. Hence, since $W_{s}^{\prime \perp}=W^{\prime \perp} \oplus \mathbb{C} s$, there must exist a $\lambda \in \mathbb{C}$ such that $\left(z^{n}-\lambda\right) s \in W^{\prime \perp}$.

Now assume that (9.2) holds, then for every $v \in W_{s}^{\prime}$ one has

$$
\left\langle z^{n} v \mid s(z)\right\rangle=\left\langle v \mid z^{n} s(z)\right\rangle=\lambda\langle v \mid s(z)\rangle=0,
$$

hence $z^{n} v \in W_{s}$.

The same proof also holds for $W^{\perp}$ and $W_{u}^{\perp}$, which proves the case of $W_{u}$.

From this we deduce:

Corollary 9.1. Let $W \in G r(H)$ be such that $L_{W}$ belongs to the $m$ vector n-constrained KP-hierarchy, i.e. there exists a subspace $W^{\prime} \subset W$ of codimension $m$ such that $z^{n} W^{\prime} \subset W$. Then $q(t) \partial q(t)^{-1}$ and $r(t)^{-1} \partial^{-1} r(t)$, for $q(t)=\left\langle\psi_{W}(t, z) \mid s(z)\right\rangle$ and $r(t)=\left\langle\psi_{W}^{*}(t, z) \mid u(z)\right\rangle$ with $s$ and $u$ satisfying (9.2), are elementary Bäcklund-Darboux transformation that preserve the $m$-vector $n$-constrained hierarchy.

\section{$\S 10$. From KP to the 1-Toda Lattice Hierarchy}

The 1-Toda lattice hierarchy, as described in [1] (see also [3] and [11]) is the set of deformation equations (Lax equations)

$$
\frac{\partial L}{\partial t_{n}}=\left[\left(L^{n}\right)_{+}, L\right]
$$

for infinite matrices

$$
L=L(t, \Lambda)=\Lambda+\sum_{i=0}^{\infty} a_{i} \Lambda^{-i} \quad \text { with } \Lambda=\sum_{k \in \mathbb{Z}} \frac{\epsilon_{k}}{\epsilon_{k+1}} E_{k, k+1},
$$


depending on $t=\left(t_{1}, t_{2}, \ldots\right)$ where $\partial_{n}=\partial / \partial t_{n}$ and where $\epsilon_{k} \in \mathbb{C}^{\times}, a_{i}=$ $\sum_{k \in \mathbb{Z}} a_{i}(k, t) E_{k k}$ is an infinite diagonal matrix. Here $A_{+}$refers to the upperdiagonal part of the matrix $A$, including the diagonal. We denote by $A_{-}=$ $A-A_{+}$. For notational covenience we write $\Lambda=\epsilon \sum_{k \in \mathbb{Z}} E_{k, k+1} \epsilon^{-1}$ with $\epsilon=$ $\sum_{k \in \mathbb{Z}} \epsilon_{k} E_{k k}$.

To the problem (10.1) one associates wavevectors $\Psi(t, z)$ and adjoint wavevectors $\Psi^{*}(t, z)$,

$$
\begin{aligned}
\Psi(t, z) & =\left(\epsilon_{n} \psi_{n}(t, z)\right)_{n \in \mathbb{Z}}=P(t, \Lambda) e^{\sum_{k=0}^{\infty} t_{k} \Lambda^{k}} \epsilon \chi(z) \\
\Psi^{*}(t, z) & =\left(\epsilon_{n}^{-1} z \psi_{n+1}^{*}(t, z)\right)_{n \in \mathbb{Z}}=\left(P(t, \Lambda)^{T}\right)^{-1} e^{-\sum_{k=0}^{\infty} t_{k} \Lambda^{-k}} \epsilon^{-1} \chi\left(z^{-1}\right)
\end{aligned}
$$

which satisfies

$$
\begin{aligned}
L \Psi(t, z) & =z \Psi(t, z), & L^{T} \Psi^{*}(t, z) & =z \Psi^{*}(t, z), \\
\partial_{n}(\Psi(t, z)) & =\left(L^{n}\right)_{+} \Psi(t, z), & \partial_{n}\left(\Psi^{*}(t, z)\right) & =\left(\left(L^{n}\right)_{+}\right)^{T} \Psi^{*}(t, z) .
\end{aligned}
$$

Here $A^{T}$ stands for the transposed of the matrix $A, \chi(z)$ is the infinite columnvector $\chi(z)=\left(z^{n}\right)_{n \in \mathbb{Z}}$ and

$$
P(t, \Lambda)=I+\sum_{k=1}^{\infty} p_{k} \Lambda^{-k} .
$$

Notice that in this situation $L=P(t, \Lambda) \Lambda P(t, \Lambda)^{-1}$. We can formulate the 1-Toda lattice hierarchy in an other way, viz. as follows (see also [1]):

Proposition 10.1. The following two statements are equivalent:

(a) The Lax operator $L$ of the form (10.2) satisfies the 1-Toda lattice hierarchy (10.1).

(b) $\psi_{n}(t, z)$ is a wavefunction of the KP-hierarchy for all $n \in \mathbb{Z}$, with $\psi_{n}^{*}(t, z)$ its adjoint wavefunction. These (adjoint) wave functions satisfy the $n-m$-th modified KP-hierarchy:

$$
\left\langle\psi_{n}(t, z) \mid \psi_{m}^{*}(s, z)\right\rangle=0 \text { for all } n \geq m .
$$

The proof of this proposition is straightforward and we omit it here.

Part (b) of the above proposition tells us that the planes $W_{n} \in G r^{(n)}(H)$ related to $\psi_{n}$ form an infinite flag, in fact one has two infinite flags

$$
\begin{aligned}
& \cdots \subset W_{n+1} \subset W_{n} \subset W_{n-1} \subset W_{n-2} \subset \cdots, \\
& \cdots \supset W_{n+1}^{\perp} \supset W_{n}^{\perp} \supset W_{n-1}^{\perp} \supset W_{n-2}^{\perp} \supset \cdots .
\end{aligned}
$$


So starting with one plane $W \in G r^{(n)}(H)$, with wavefunction $\psi_{W}(t, z)$, adjoint wavefunction $\psi_{W}^{*}(t, z)$ and tau-function $\tau_{W}$, we can apply recursively BäcklundDarboux transformations and adjoint Bäcklund-Darboux transformations to construct a whole flag of spaces. To be more precise:

Proposition 10.2. Let $W \in G r^{(n)}(H)$, with wavefunction $\psi_{W}(t, z)$, adjoint wavefunction $\psi_{W}^{*}(t, z)$ and tau-function $\tau_{W}$ and let $w_{n}, w_{n+1}, w_{n+2}, \ldots$ be an ordered basis of $W$ and let $w_{n-1}^{\dagger}, w_{n-2}^{\dagger}, w_{n-3}^{\dagger}, \ldots$ be an ordered basis of $W^{\perp}$, then the spaces

$$
W_{m}= \begin{cases}\left\{w \in W \mid\left\langle w \mid w_{k}\right\rangle=0 \text { for } k<m\right\} & \text { for } m>n, \\ W & \text { if } n=m \\ W \oplus \sum_{k=m}^{n-1} \mathbb{C} w_{k}^{\dagger} & \text { for } m<n\end{cases}
$$

and spaces $W_{m}^{\perp}$ form infinite flags of the form (10.7). The corresponding wavefunctions $\psi_{m}(t, z)$, adjoint wavefunction $\psi_{m}^{*}(t, z)$ and tau-function $\tau_{m}$ are equal to

$$
\psi_{m}=\left\{\begin{array}{rr}
\mathcal{W}\left(\psi_{W} ; w_{n}, \ldots, w_{m-1} ; \psi_{W}^{*} ; \emptyset\right)^{-1} & \\
\quad \times \mathcal{W}\left(\psi_{W} ; w_{n}, \ldots, w_{m-1} ; \psi_{W}^{*} ; \emptyset ; \psi_{W}\right) & \text { for } m>n \\
\psi_{W} & \text { if } n=m, \\
\mathcal{W}\left(\psi_{W} ; \emptyset ; w_{m}^{\dagger}, \ldots, w_{n-1}^{\dagger}\right)^{-1} & \\
\quad \times \mathcal{W}\left(\psi_{W} ; \emptyset ; \psi_{W}^{*} ; w_{m}^{\dagger}, \ldots, w_{n-1}^{\dagger} ; \psi_{W}\right) & \text { for } m<n
\end{array}\right.
$$

and

$$
\begin{aligned}
& \psi_{m}^{*}=\left\{\begin{array}{cc}
(-)^{n+m+1} \mathcal{W}\left(\psi_{W}^{*} ; \emptyset ; \psi_{W} ; w_{n}, \ldots, w_{m-1}\right)^{-1} & \\
\quad \times \mathcal{W}\left(\psi_{W}^{*} ; \emptyset ; \psi_{W} ; w_{n}, \ldots, w_{m-1} ; \psi_{W}^{*}\right) & \text { for } m>n, \\
\psi_{W}^{*} & \text { if } n=m, \\
(-)^{n+m+1} \mathcal{W}\left(\psi_{W}^{*} ; w_{m}^{\dagger}, \ldots, w_{n-1}^{\dagger} ; \psi_{W} ; \emptyset\right)^{-1} & \\
\quad \times \mathcal{W}\left(\psi_{W}^{*} ; w_{m}^{\dagger}, \ldots, w_{n-1}^{\dagger} ; \psi_{W} ; \emptyset ; \psi_{W}^{*}\right) & \text { for } m<n
\end{array}\right. \\
& \tau_{m}= \begin{cases}\mathcal{W}\left(\psi_{W} ; w_{n}, \ldots, w_{m-1} ; \psi_{W}^{*} ; \emptyset\right) \tau_{W} & \text { for } m>n, \\
\tau_{W} & \text { if } n=m, \\
\mathcal{W}\left(\psi_{W} ; \emptyset ; \psi_{W}^{*} ; w_{m}^{\dagger}, \ldots, w_{n-1}^{\dagger}\right) \tau_{W} & \text { for } m<n,\end{cases}
\end{aligned}
$$

Moreover, $\Psi(t, z)=\left(\epsilon_{n} \psi_{n}(t, z)\right)_{n \in \mathbb{Z}}$ is a wavevector and $\Psi^{*}(t, z)=$ $\left(\epsilon_{n}^{-1} z \psi_{n+1}^{*}(t, z)\right)_{n \in \mathbb{Z}}$ is its adjoint wavevector of the 1-Toda lattice hierarchy.

The proof of this proposition is straightforward. 
As an application of the above, we consider certain orthogonal polynomials that are related to Matrix models, see e.g. [3], [11] and references therein.

Let $(a, b)$ be an interval of $\mathbb{R}(a=-\infty$ and $b=\infty$ is allowed $)$ and $w(y)$ a weight function which is non-negative. With these data we associate the inner product

$$
\left(f_{1} \mid f_{2}\right)=\int_{a}^{b} f_{1}(y) f_{2}(y) w(y) d y
$$

which is well defined for all functions $f$ for which $\sqrt{w} f$ is quadratically integrable on $(a, b)$.

The numbers

$$
c_{n}=\int_{a}^{b} y^{n} w(y) d y,
$$

are called the moments of the weight function $w$. It is well-known (see e.g. [6]) that the polynomials

$$
p_{n}(y)=\frac{\operatorname{det}\left(\begin{array}{cccc}
c_{0} & c_{1} & \cdots & c_{n} \\
c_{1} & c_{2} & \cdots & c_{n+1} \\
\vdots & \vdots & \cdots & \vdots \\
c_{n-1} & c_{n} & \cdots & c_{2 n-1} \\
1 & y & \cdots & y^{n}
\end{array}\right)}{\operatorname{det}\left(c_{i+j}\right)_{0 \leq i, j \leq n-1}}
$$

form an orthogonal set, i.e.,

$$
\left(p_{m} \mid p_{n}\right)=\delta_{m n} \frac{\operatorname{det}\left(c_{i+j}\right)_{0 \leq i, j \leq n}}{\operatorname{det}\left(c_{i+j}\right)_{0 \leq i, j \leq n-1}} .
$$

Now consider the generating series of the moments

$$
f(z)=\sum_{k=0}^{\infty} c_{k} z^{-k-1}
$$

and let

$$
f_{i}(z)=\sum_{k=0}^{\infty} c_{k} z^{i-k-1} .
$$

Notice that

$$
-f(z)=\int_{a}^{b} \frac{w(y) d y}{y-z}
$$


is the Stieltjes transform. Now we consider the following chain of subspaces of $H_{+}$:

$$
W_{0}=H_{+} \quad \text { and } W_{n}=\left\{w \in H_{+} \mid\left\langle w \mid f_{i}\right\rangle=0 \text { for } i=0,1, \ldots, n-1\right\} .
$$

Since $\left(y^{j}, p_{m}(y)\right)=0$ for $j<m$, we get that (cf. [11])

$$
W_{m}=\text { the closure of } \operatorname{Span}\left\{p_{k} \mid k \geq m\right\} .
$$

Next we compute the tau-function corresponding to this chain. We have

$$
\begin{aligned}
\left\langle\frac{\partial^{p} e^{\sum_{k} t_{k} z^{k}}}{\partial x^{p}} \mid f_{q}(z)\right\rangle & =\left\langle z^{p+q} e^{\sum_{k} t_{k} z^{k}} \mid f_{0}(z)\right\rangle \\
& =\int_{a}^{b} z^{p+q} e^{\sum_{k} t_{k}(z)^{k}} w(z) d z \\
& =c_{p+q}(t),
\end{aligned}
$$

where $c_{n}(t)$ is the $n$-th moment of the $t$-dependent inner product (cf. (10.13))

$$
\left(f_{1}(t, z) \mid f_{2}(t, z)\right)_{t}=\int_{a}^{b} f_{1}(t, z) f_{2}(t, z) e^{\sum_{k} t_{k}(z)^{k}} w(z) d z .
$$

We find that the to $W_{n}$ corresponding wave function $\psi_{n}(t, z)=$ $P_{n}(t, z) e^{\sum_{k} t_{k} z^{k}}$ is equal to

$$
P_{n}(t, z)=\frac{\operatorname{det}\left(\begin{array}{cccc}
c_{0}(t) & c_{1}(t) & \cdots & c_{n}(t) \\
c_{1}(t) & c_{2}(t) & \cdots & c_{n+1}(t) \\
\vdots & \vdots & \cdots & \vdots \\
c_{n-1}(t) & c_{n}(t) & \cdots & c_{2 n-1}(t) \\
1 & z & \cdots & z^{n}
\end{array}\right)}{\operatorname{det}\left(c_{i+j}(t)\right)_{0 \leq i, j \leq n-1}} .
$$

These $P_{n}(t, z)$ form the orthogonal polynomials with respect to the $t$-dependent inner product $(\cdot \mid \cdot)_{t}$, defined by (10.18). Moreover, the $\psi_{n}$ 's for $n=0,1,2, \ldots$ form a set of 1-Toda wavefunctions and the $\tau_{n}$ 's where

$$
\tau_{n}(t)=\operatorname{det}\left(c_{i+j}(t)\right)_{0 \leq i, j \leq n-1},
$$

form a set of 1-Toda tau-functions.

For all $w_{n} \in W_{n}$ we have that $\left\langle w_{n} \mid f_{i}\right\rangle=0$ for $i=0,1, \ldots, n-1$, then $\left\langle z w_{n} \mid f_{i}\right\rangle=\left\langle w_{n} \mid z f_{i}\right\rangle=\left\langle w_{n} \mid f_{i+1}\right\rangle$ is zero for $i=0,1, \ldots, n-2$. Hence $z W_{n} \subset$ 
$W_{n-1}$ and we deduce that $\psi_{n}(t, z)$ belongs to the 1-vector 1-constrained KP hierarchy, i.e.,

$$
L_{W_{n}}=\partial+q_{n} \partial^{-1} r_{n}
$$

with $q_{n}$ and $r_{n}$ eigenfunctions, respectively adjoint eigenfunctions of $L_{W_{n}}$. From the construction in Section 3 of [12], it is easy to determine the $q_{n}$ and $r_{n}$ 's, in fact

$$
L_{W_{n}}=\partial+g_{n} \frac{\tau_{n+1}}{\tau_{n}} \partial^{-1} \frac{\tau_{n-1}}{\tau_{n}}
$$

with $g_{n} \in \mathbb{C}^{\times}$. Hence

$$
\begin{aligned}
z \psi_{n} & =\frac{\partial \psi_{n}}{\partial x}+g_{n} \frac{\tau_{n+1}}{\tau_{n}} \partial^{-1} \frac{\tau_{n-1}}{\tau_{n}}\left(\psi_{n}\right) \\
& =\frac{\partial \psi_{n}}{\partial x}+g_{n} \frac{\tau_{n+1}}{\tau_{n}} \partial^{-1} \frac{\tau_{n-1}}{\tau_{n}} \frac{\tau_{n}}{\tau_{n-1}} \partial \frac{\tau_{n-1}}{\tau_{n}}\left(\psi_{n-1}\right) \\
& =\frac{\partial \psi_{n}}{\partial x}+g_{n} \frac{\tau_{n+1} \tau_{n-1}}{\left(\tau_{n}\right)^{2}} \psi_{n-1} .
\end{aligned}
$$

Since also

$$
\psi_{n+1}=\frac{\partial \psi_{n}}{\partial x}-\frac{\partial}{\partial x}\left(\log \frac{\tau_{n+1}}{\tau_{n}}\right) \psi_{n}
$$

we find that

$$
z \psi_{n}=\psi_{n+1}+\frac{\partial}{\partial x}\left(\log \frac{\tau_{n+1}}{\tau_{n}}\right) \psi_{n}+g_{n} \frac{\tau_{n+1} \tau_{n-1}}{\left(\tau_{n}\right)^{2}} \psi_{n-1} .
$$

We can now determine the constant $g_{n}$. For this purpose we take $\left\langle z \psi_{n} \mid f_{n-1}\right\rangle$. Then from (10.21) we deduce that

$$
g_{n}=\frac{\left\langle\psi_{n} \mid f_{n}\right\rangle \tau_{n}}{\left\langle\psi_{n-1} \mid f_{n-1}\right\rangle \tau_{n-1}} \frac{\tau_{n}}{\tau_{n+1}}=\frac{\tau_{n+1}}{\tau_{n}} \frac{\tau_{n}}{\tau_{n+1}}=1
$$

Thus we find that the $P_{n}(t, z)$ satisfy the following recursion relation:

$$
z P_{n}(t, z)=P_{n+1}(t, z)+\frac{\partial}{\partial x}\left(\log \frac{\tau_{n+1}}{\tau_{n}}\right) P_{n}(t, z)+\frac{\tau_{n+1} \tau_{n-1}}{\tau_{n}^{2}} P_{n-1}(t, z) .
$$




\section{References}

[1] Adler, M., Horozov, E. and van Moerbeke, P., The solution to the $q$-KdV equation, Phys. Lett. A, 242 (1998), 139-151.

[2] Adler, M. and van Moerbeke, P., Birkhoff Strata, Bäcklund Transformations and Regularization of Isospectral Operators, Adv. in Math., 108 (1994), 140-204.

[3] - Matrix integrals, Toda symmetries, Virasoro constraints and orthogonal polynomials, Duke Math. J., 80 (1995) 23-88.

[4] Aratyn, H., Nissimov, E. and Pacheva, S., Method of squared eigenfunction potentials in integrable hierarchies of KP type, Comm. Math. Phys., 193 (1998), 493-525 (solv-int 9701017).

[5] Bakalov, B., Horozov, E. and Yakimov, M., Bäcklund-Darboux transformations in Sato's Grassmannian, Serdica Math. J., 22 (1996), 571-586.

[6] Bateman, H., Higher transcendental functions, Vol II, McCraw-Hill, New York, 1953.

[7] Chau, L.-L., Shaw, J. C. and Yen, H. C., Solving the KP hierarchy by gauge transformations, Comm. Math. Phys., 149 (1992), 263-278.

[8] Crum, M. M., Associated Sturm-Liouville systems, Quart. J. Math., 6 (1955), 121-127.

[9] Date, E., Jimbo, M., Kashiwara, M. and Miwa, T., Transformation groups for soliton equations, in: Nonlinear integral systems - classical theory and quantum theory, M. Jimbo and T. Miwa, eds., World Scientific, 39-120, 1983.

[10] Dickey, L. A., On the constrained KP hierarchy. II, Lett. Math. Phys., 35 (1995), 3, 229-236.

[11] Haine, L. and Horozov, E., Toda orbits of Laguere polynomials and representations of the Virasoro algebra, Bull. Sci. Math., 117 (1993), 485-518.

[12] Helminck, G. F. and van de Leur, J. W., An analytic description of the vector constrained KP hierarchy, Comm. Math Phys., 193 (1998), 627-641.

[13] - Constrained and Rational Reductions of the KP hierarchy, in: Supersymmetry and Integrable Models, Springer Lecture Notes in Phys., 502 (1998), 167-182.

[14] Kac, V. G. and van de Leur, J. W., The $n$-component $K P$ hierarchy and representation theory, in Important developments in soliton theory, A. S. Fokas and V. E. Zakharov eds., Springer Ser. Nonlinear Dynam., (1993), 302-343. Extended version of this paper with the same name will appear in the second edition of this book.

[15] Kasman, A., Darboux Transformations from n-KdV to KP, Acta Appl. Math., 49 (1997), 179-197.

[16] Krichever, I., General rational reductions of the KP hierarchy and their symmetries, Funct. Anal. Appl., 29 (1995), 75-80.

[17] Matveev, V. B. and Salle, M. A., Darboux Transformations and Solitons, Springer Ser. Nonlinear Dynam., Springer, Berlin, 1991.

[18] Mulase, M., Algebraic theory of the KP equations, in Interface between Mathematics and physics, S. T. Yau ed., International Press Company (1994), 157-223.

[19] Oevel, W., Darboux theorems and Wronskian formulas for integrable systems (I. Constrained KP flows), Phys. A, 195 (1993), 533-576.

[20] Oevel, W. and Schief, W., Darboux theorems and the KP hierarchy, in: Applications of analytic and geometric Methods in Differential Equations, Proceedings of the NATO Advanced Research Workshop, Exeter, 14-17 July 1992, UK, P. A. Clarkson ed., Kluwer Publ. (1993), 193-206.

[21] Oevel, W. and Strampp, W., Wronskian solutions of the constrained KadomtsevPetviashvili hierarchy, J. Math. Phys., 37 (1996), 6213-6219.

[22] Pressley, A. and Segal, G., Loop groups, Oxford Math. Monogr., Oxford University Press, Oxford, 1988.

[23] Segal, G. and Wilson, G., Loop groups and equations of KdV type, Publ. Math IHES, 63 (1985), 1-64.

[24] Shiota, T., Prym varieties and soliton equations, in: Infinite-dimensional Lie algebras and groups, Luminy-Marseille, 1988, 407-448, Adv. Ser. Math. Phys., 7, World Sci. Publishing, Teaneck, NJ, 1989. 
[25] Wilson, G., Commuting flows and conservation laws for Lax equations, Math. Proc. Cambridge Philos. Soc., 86 (1979), 131-143. 